\title{
A self-validating digital coriolis mass-flow meter: an overview
}

\author{
M.P. Henry, D.W. Clarke, N. Archer, J. Bowles, M.J. Leahy ${ }^{\dagger}$, R.P. Liu, J. Vignos ${ }^{\ddagger}$, F.B. Zhou.
}

Engineering Science Department, Oxford University, Parks Road, Oxford OX1 3PJ, UK. $\dagger$ PEI Technologies, University of Limerick, Ireland.

$\$$ The Foxboro Company, 33 Commercial Street, Foxboro, MA02035, USA.

\section{Corresponding Author: manus.henry@eng.ox.ac.uk}

\begin{abstract}
A new implementation of a Coriolis mass-flow meter transmitter is described. It is based on digital components, and has improved performance compared with the commercial, mostly analogue, transmitter using the same flowtube (transducer). Improvements are found in flowtube control, measurement precision, and performance with two-phase and partially-empty conditions, including batching from empty. The new transmitter is viewed as a second-generation Sensor Validation (SEVA) demonstrator, in which experience from validating the commercial analogue transmitter has led to a redesign using digital technology. The resulting SEVA transmitter provides improved measurement performance and reduced vulnerability to fault conditions, as well as online estimates of measurement quality and fault compensation (Henry and Clarke, 1993).
\end{abstract}

Keywords: Coriolis mass-flow metering; Sensor validation; Non-linear control; Intelligent sensors; Field Programmable Gate Arrays; Hardware Compilation.

\section{Introduction}

Coriolis mass-flow metering has been used in industry since the early 80's. A Coriolis meter (Figure 1) consists of a vibrating flowtube and an electronic transmitter to maintain the vibrations and to perform measurement calculations. Flowtube designs vary widely (e.g. straight-through pipes, Utubes, B-tubes, with single path or split flow configurations), but the basic operating principle remains the same: mass-flow moving through a vibrating tube causes a proportional Coriolis force to act, which is detected as a phase difference between two sensors. Raszillier and Durst (1991) describe the physical background for Coriolis mass-flow metering, while Cascetta, Cignolo, Goria, Martini, Rivetti \& Vigo (1992) give a comparison of the performance of seven commercial mass-flow meters.

Almost all transmitters are designed to vibrate the flowtube at its natural frequency; this frequency is a function of the process fluid density, which can thus be calculated and given as an additional measurement. The process fluid temperature is also monitored, to provide temperature correction of the mass-flow and density (compensating for tube stiffness), and as an extra process measurement. Further variables may be provided e.g. the ratio of mass-flow and density yields the volumetric flow rate. Thus the Coriolis meter is a fairly sophisticated example of a multi-variable industrial instrument.
Coriolis metering offers several advantages over other flowmeter technologies. The direct measurement of mass-flow is useful in applications where, for example energy content, batch size or product value is a function of mass rather than volume. Repeatability of $0.1 \%$ or better, and a typical turndown (i.e. the ratio between maximum and minimum flow rates at which the accuracy specification is achieved) of at least 20:1 compare well with other flow meters (Cascetta et al., 1992). Finally, Coriolis meters can deal with difficult, nonNewtonian fluids such as slurries and foodstuffs.

There are also disadvantages and limitations. The meters are expensive to purchase and install. Most industrial instrumentation uses a single pair of wires for both power and communications. In the predominant $4-20 \mathrm{~mA}$ regime, two-wire operation

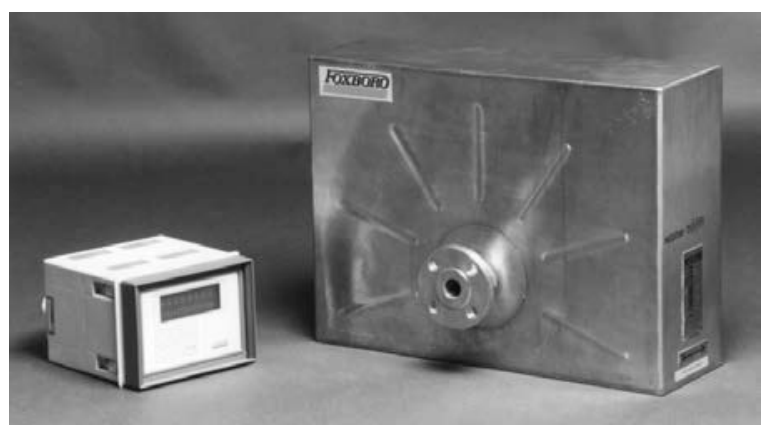

Fig. 1. A commercial Coriolis mass-flow meter, with transmitter (left) and flowtube (right) 
places exacting constraints on the power budget of an instrument, and for commercial Coriolis meters the current needed to drive the flowtube (typically 10$100 \mathrm{~mA}$ ) must be provided by an additional power supply, adding to installation costs.

Other concerns are the ability to deal with external vibration, flow pulsation (Cheesewright and Clark, 1998) and, for thin-walled designs, the threat of process fluid leakage via erosion or corrosion. Perhaps the most serious limitation, however, is the impact of two-phase (gas-liquid) flow (Skea and Hall, 1999). Even a short burst of gas in a liquid flow stream (due for example to cavitation) may disrupt flowtube oscillation. Similarly many designs are unable to operate in a partially empty condition. There are numerous applications in which Coriolis metering would be highly desirable (e.g. tanker loading and emptying), but where transient air (e.g. at the start and/or end of a batch) makes this difficult or impossible.

The Engineering Science Department at the University of Oxford has been carrying out research into Self-Validating Sensors (Henry and Clarke, 1993, Henry, 1994) since 1988, with funding from industry and the UK government. The self-validating or SEVA sensor model, developed at Oxford, is now being considered as a UK national standard for the reporting of measurement quality in industrial sensors (SIRA, 1998; BSI, 1998). It assumes the availability of internal computing power for selfdiagnostics based on the designer's expertise, and of digital communications to convey measurement, diagnostic and maintenance data (Henry 1995b). The SEVA model proposes a generic set of metrics for describing measurement quality, independent of the underlying sensor technology. The key metric is the on-line uncertainty of each measurement. This permits generic strategies for responding to changes in measurement quality at the control system level. Many of the basic SEVA concepts were developed through consideration of the Coriolis meter (Henry 1995a), and in particular the impact of a single fault condition on the quality of each measurement.

This paper is arranged as follows:

- The design and operation of an analogue transmitter and flowtube

- Initial validation work and the development of the digital transmitter concept

- Hardware and software implementation

- Flowtube oscillation control

- Measurement algorithms

- Results for batching from empty

- Two-phase flow performance

- Conclusions and future research

More detailed discussion of several of these topics will be the subject of future publications.

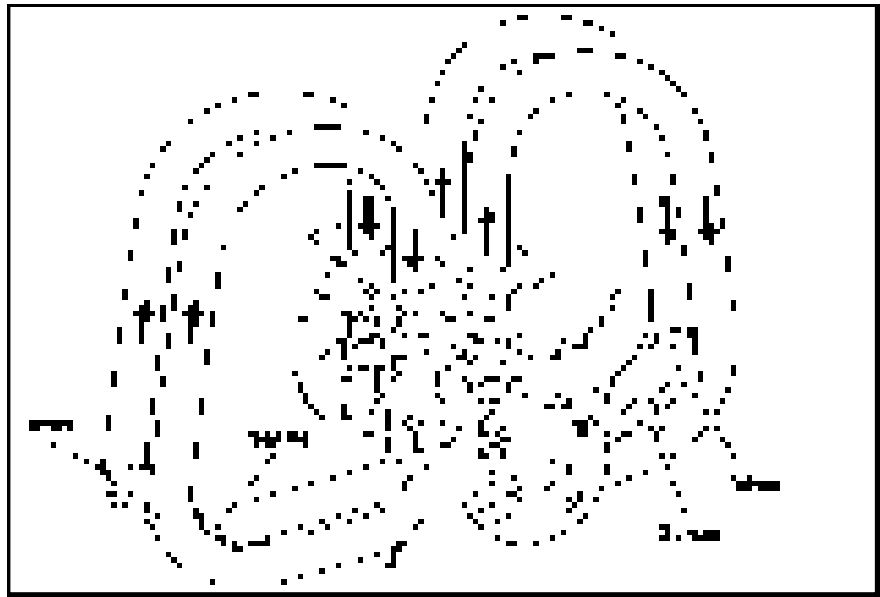

Fig. 2. Flowtube (B-tube) with drivers and sensors

\section{Flowtube and Analogue Transmitter Design}

This section provides an overview of the design and operation of the Coriolis flowtube and the functions carried out by the analogue transmitter.

Figure 2 shows the flowtube used in the research. It consists of a set of mounting flanges for connection to the process pipeline, and a central manifold block supporting two parallel planar B-shaped loops that are oriented orthogonally to the pipeline. The manifold connects the loops serially, so that all the flow travels through first one loop and then the other (some other flowtube designs have split-flow configurations). An electromagnetic driver and a velocity sensor are attached between each end of the loops. The drivers are energised with currents of equal magnitude but opposite sign (i.e. $180^{\circ}$ out of phase) to cause the straight section of each loop to rotate about its midpoint. Applying a sinusoidal drive current causes each straight section to undergo oscillatory motion about the axis of symmetry of the loop. During normal operation, a $25 \mathrm{~mm}$ diameter flowtube with a $60 \mathrm{~cm}$ straight section vibrates with a typical maximum excursion of about $1.5 \mathrm{~mm}$.

The drivers are excited to vibrate at the natural frequency of oscillation of the flowtube $\omega$, typically 80 Hertz. At any instant, each straight section experiences an angular velocity $\boldsymbol{\Omega}$ given by

$$
\Omega=\Omega_{0} \sin \omega t
$$

Flow at velocity $\mathbf{v}$ experiences a Coriolis acceleration $2 \Omega \times \mathbf{v}$; this is manifest as a phase difference between the sinusoidal sensor outputs. Typically at maximum flow rate a $4^{\circ}$ phase shift is observed.

Figure 3 shows an overview of the hardware design of the commercial analogue transmitter. Sensor voltages $\mathrm{V}_{\mathrm{A} 0}$ and $\mathrm{V}_{\mathrm{B} 0}$ are approximately $180^{\circ}$ out of phase. Each is amplified; a variable gain on $V_{\mathrm{B} 0}$ ensures that the resulting $V_{B 1}$ has the same amplitude as $\mathrm{V}_{\mathrm{A} 1}$. These scaled signals are summed and differenced. The difference, $\mathrm{V}_{\mathrm{DRV}}$ (drive signal) is 


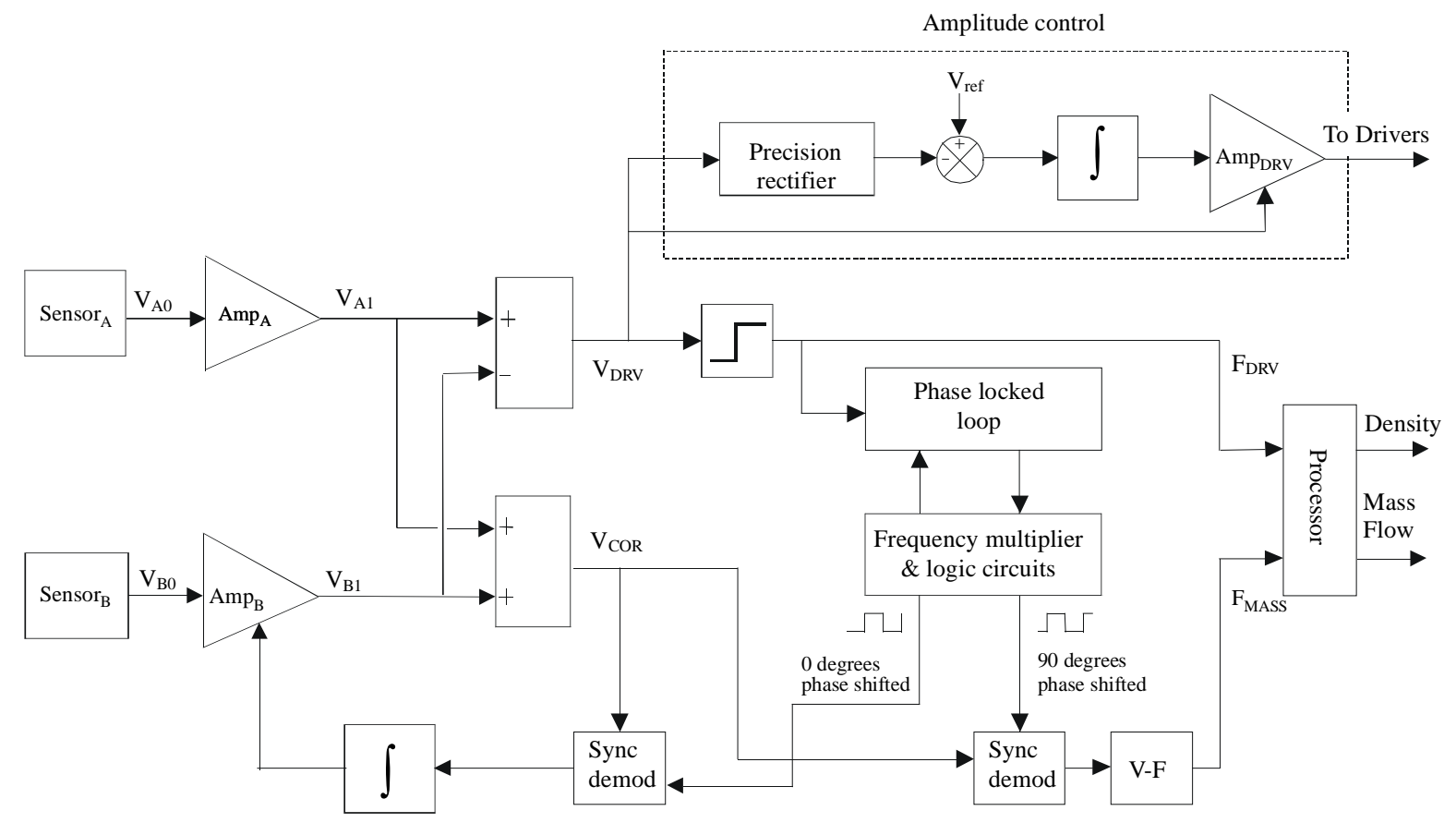

Fig. 3. Schematic of analogue transmitter hardware

proportional to the amplitude of oscillation of sensor $A$ with phase at the mid-point between the phases of sensors A and B. The sum, $\mathrm{V}_{\mathrm{COR}}$ (Coriolis signal) has an amplitude proportional to the phase difference (offset by $180^{\circ}$ ) between sensors $\mathrm{A}$ and $\mathrm{B}$, and is 90 degrees out of phase with $\mathrm{V}_{\mathrm{DRV}}$.

The two signals $\mathrm{V}_{\mathrm{COR}}$ and $\mathrm{V}_{\mathrm{DRV}}$ are used by the analogue transmitter to carry out several key tasks, described in the following subsections.

\subsection{Flowtube oscillation frequency generation}

The signal $\mathrm{V}_{\mathrm{DRV}}$ is squared using a comparator circuit to give $F_{D R V}$, which is read by the processor as an estimate of the flowtube oscillation frequency. $F_{D R V}$ is also fed into a phase-locked loop (Horowitz and Hill, 1989) to provide gating signals for synchronous demodulation (Bergovic Djuric, Dunlap \& Phadke, 1993).

\subsection{Phase difference generation}

The Coriolis signal $\mathrm{V}_{\mathrm{COR}}$ is composed primarily of a sinusoid with amplitude proportional to the phase difference between the two sensors. It is however liable to contamination by noise caused by, for example, the presence of higher harmonics. Synchronous demodulation is used to eliminate all components except those with a desired frequency and phase. The Coriolis component of the signal has the same frequency but $90^{\circ}$ phase offset from $V_{D R V}$. A suitable gating signal is generated by circuitry fed from the phase-locked loop. The result of synchronous demodulation is a signal proportional to the amplitude of $\mathrm{V}_{\mathrm{COR}}$ i.e. to phase difference and hence to mass-flow. A voltage to frequency converter generates a square wave signal $\mathrm{F}_{\text {MASS }}$ which is sent to the processor.

\subsection{Sensor balance}

The sum and difference operations assume that the amplitudes of $\mathrm{V}_{\mathrm{A} 1}$ and $\mathrm{V}_{\mathrm{B} 1}$ are the same, for which purpose a variable gain is provided for $\mathrm{Amp}_{\mathrm{B}}$. This gain is adjusted to minimise a component in $\mathrm{V}_{\mathrm{COR}}$ proportional to the amplitude of $\mathrm{V}_{\mathrm{A} 1}-\mathrm{V}_{\mathrm{B} 1}$, which is extracted using synchronous demodulation where the gating signal is in phase with $\mathrm{V}_{\mathrm{DRV}}$.

\subsection{Drive signal generation and amplitude control} As the flowtube has a high Q (typically 2000), only a narrow range of frequencies will cause the flowtube to resonate. To ensure a suitable drive frequency is generated, positive feedback is used: the frequency and (midpoint) phase from the sensors, provided by $\mathrm{V}_{\mathrm{DRV}}$, is sent to the drivers. The signal is first multiplied by a suitable gain (in AmpDrv ). This gain is selected via negative feedback which acts to maintain a constant amplitude of oscillation. $\mathrm{V}_{\text {ref }}$, the amplitude setpoint, is $0.3 \mathrm{~V}$.

Providing the required drive gain is essential for the maintenance of flowtube oscillation. In particular, if damping is high (caused for example by an aerated fluid), and the maximum drive gain is reached (due to saturation in Amp ${ }_{D R V}$ ), then flowtube stalling occurs instantaneously. Insufficient current is sent to the drivers, which causes a drop in oscillation amplitude, which in turn causes a further lowering of output current: the net result is that vibration ceases.

The actions of the amplitude control and sensor balance circuitry together ensure that normally the amplitude of the Sensor A signal is maintained at $0.3 \mathrm{~V}$, while the amplitude of Sensor B varies (typically by only a few percent) for different flowtubes and with changing operating conditions. 


\subsection{Processor tasks}

The processor converts the signals $F_{D R V}$ and $F_{M A S S}$ into engineering units, using data from a temperature measurement channel (not shown). A variety of other standard tasks are also carried out, such as keypad, display and communications handling, alarm logic, zero calibration and batch totalisation.

\section{Towards a digital transmitter}

The first SEVA prototype developed at Oxford University was based on the Coriolis meter. It consisted of the flowtube and analogue transmitter with a PC (Figure 4). Some dozen signals from the transmitter were sampled to provide measurement and diagnostic data. Control signals were generated to manipulate the operation of the transmitter. The PC and the modified transmitter together exemplified how a SEVA device would behave (Henry, 1995c).

The prototype demonstrated the detection and correction of several fault modes, as well as the generation of SEVA metrics. However, technology transfer to the commercial transmitter was not straightforward. This was partly due to code space and computing power limitations. There were also concerns about the analogue design. The ability of the processor to modify the behaviour of the analogue circuitry (regulating flowtube oscillation and generating the raw measurement data), say in response to diagnostic information, is very limited. Research showed that some fault modes were due to the behaviour of the analogue circuitry in conditions not envisaged by the designers. For example the chief cause of flowtube stalling is saturation of the drive amplifier (AmpDRV in Figure 3). In other cases, it was difficult to separate design limitations from the 'real' fault effect. For example, is the response of the meter to two-phase flow primarily a function of the physics of air bubbles in a vibrating tube, or of the electronics dealing with low signal levels?

One benefit of carrying out a sensor validation analysis is that it leads to redesign. The identification of the major limitations and fault modes of an instrument, and their impact on measurement quality, provides strong motivation for improvements in the basic design (or indeed manufacturing, installation, characterisation, and/or operating procedures where weaknesses are revealed). The idea of an all-digital transmitter design emerged in response to this first round of validation activity, and is illustrated in Figure 5.

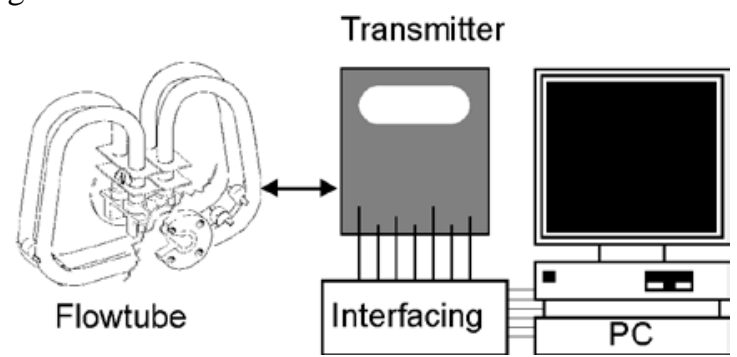

Fig. 4. Validation of the analogue transmitter

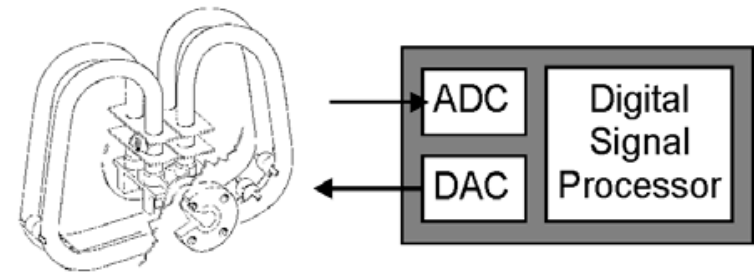

Flowtube

Digital Transmitter

Fig. 5. Digital Coriolis Transmitter

The primary intention was to replace all the analogue circuitry (other than essential front-end op-amps) with a small number of digital components, and to carry out almost all functionality within software. The expected benefits of an all-digital design were as follows:

- Provision of more computing power and memory.

- Better flowtube control through the use of a nonlinear control algorithm.

- Better measurement precision through high bandwidth, high precision data and digital processing.

- Greater operational flexibility, leading to e.g. better robustness to process aeration.

- Reduced influence of transmitter hardware on measurement data, allowing new research into the effects of two-phase flow.

- Better integration of diagnostic reasoning with flowtube operation in a single software framework.

An all-digital design seemed attractive not only for research purposes, but also for commercial implementation. One of the main reasons for the expense of Coriolis transmitters is the requirement for high-quality, high-component count, bespoke analogue circuit designs produced in low volume. By contrast, digital technology is available for mainstream consumer markets such as mobile phones in large volumes at low per unit cost. For example a 180MFlop processor is, at the time of writing, being sold for $\$ 10$ (Analog Devices, 1998), while $\$ 8$ buys a 20 bit codec (two channels each of ADC and DAC, operating at audio bandwidth: Texas Instruments, 1998). These two components cover about $90 \%$ of digital Coriolis transmitter functionality. The commercial appeal is clear: a design employing a low component count and a high software content, based on rapidly evolving, competitive, mainstream technology.

The primary challenge at the outset of the work was to determine whether digital technology could deliver the required measurement performance. For example, a typical frequency of oscillation is $80 \mathrm{~Hz}$, and at full flow the observed phase shift is typically 4 degrees. For a 100:1 turndown ratio, and 0.1\% repeatability, this translates to a timing resolution of 1.4 nanoseconds or, assuming a $44 \mathrm{kKz}$ sampling rate, resolving phase difference to 0.0006 of a sample 
interval (in practice averaging over a number of cycles eases this requirement a little). In addition, audio-quality components, unlike conventional instrumentation devices, are not designed with DC stability and amplitude precision as high priorities, potentially leading to measurement bias.

A series of prototypes have been built at Oxford, and several analogue transmitter limitations have been overcome. Benefits include:

- Improved measurement precision.

- Improved flowtube control, especially during start-up and in response to two-phase flow.

- Techniques for on-line monitoring and reduction of ADC zero drift and gain imbalance.

- Repeatable, monotonic measurement errors in response to two phase flow: results for batching from empty show large improvements.

- Research into dynamic performance of Coriolis meters, particularly in response to external vibration.

Five prototype transmitters are currently on trial in paper, chemical and food processing plant, to evaluate these performance improvements under real industrial plant conditions.

\section{PROTOTYPE IMPLEMENTATION}

Figure 5 shows a digital transmitter implementation used for industrial trials. It is a modular design, based on the transputer TRAM standard, incorporating the Valcard architecture developed at Oxford for rapid prototyping of sensor validation systems (Henry 1995c; Henry, Archer, Attia, Bowles, Clarke, Fraher, page, Randall \& Yang et al. 1996; Page 1998).

The key modules are labelled as follows:

- PPC. This is a Power PC processor which performs most transmitter calculations. A commercial module, it uses a transputer as a

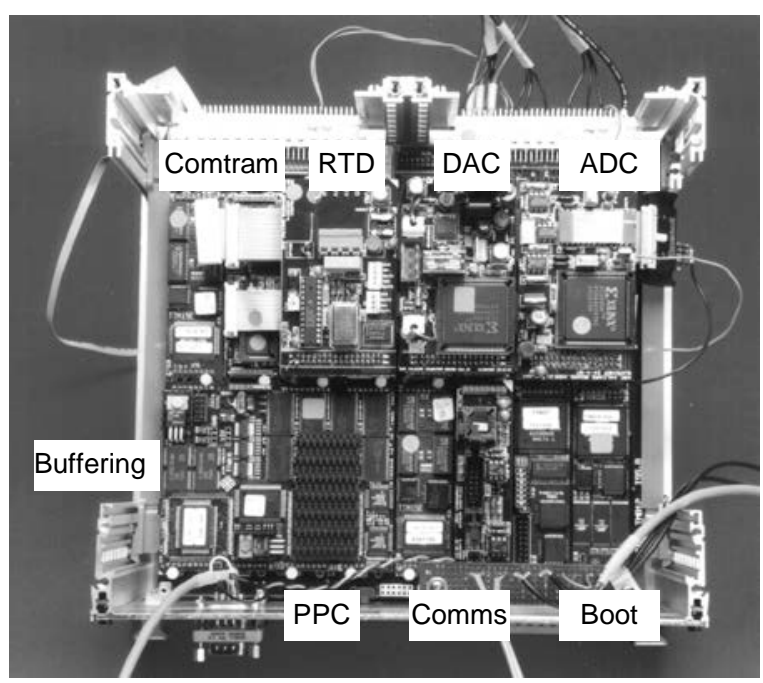

Fig. 5. Digital transmitter implementation using Valcard modules front end to enable this powerful processor to be integrated seamlessly into a transputer environment.

- Comms. These two modules provide communications with a laptop PC acting as a Hand Held Terminal, and with Foxboro's Intelligent Automation control system via a 100 parameter interface using the Modbus Plus protocol.

- Boot. A boot PROM enables standalone selfbooting. It is also possible to boot from a laptop PC, allowing rapid code modification and debugging when required.

- Buffering. Buffering is provided between the real-time data acquisition subsystem described below and the single-threaded Power PC code.

- Comtram. The Valcard architecture (Henry et al., 1996) provides for data exchange between a transputer network and a chain of FPGA modules. The FPGAs provide blocks of configurable logic to carry out arbitrary digital functions and are programmed using the Handel language (Henry, 1995c). Each FPGA module supports a daughtercard with applicationspecific circuitry. The Comtram module provides a bridge between a transputer link and two eight-bit parallel buses between the FPGA modules. There are three FPGA modules in the transmitter, as follows:

- $\quad$ RTD. This module drives the RTD temperature sensor in the flowtube, and provides data on RTD and transmitter temperature as well as 4 lines each of digital input and output.

- ADC. This module supports an AD1879 stereo, 18-bit $44 \mathrm{kHz}$ audio quality sigma-delta analogue-to-digital converter (Analog Devices), which samples the velocity sensor signals.

- DAC. This module supports an AD1865 stereo, 18-bit $44 \mathrm{kHz}$ audio quality sigma-delta digitalto-analogue converter (Analog Devices), which generates the drive output signals. It also provides MAX532 multiplying DACs (Maxim) for selecting the drive gain, as described below.

The Valcard architecture has been used to allow a process of continuous design refinement. At the outset, it was not possible to draw up a definitive working design; indeed, basic design principles were unresolved. For example, should positive feedback be used to generate the drive signals, as employed in the analogue transmitter, or should the signals be synthesised from pure sine waves of desired amplitude, frequency and phase in software? Given the 2 orders of magnitude in the working range of the drive gain, would the resolution of an 18-bit DAC be adequate both for waveform and gain, or should separate multiplying DACs be used to provide an additional gain stage? The modular structure and the ability to program the FPGAs to interface to arbitrary components has allowed design refinements in response to experimental observations. The Valcard architecture has proved an adaptable prototyping 
platform for complex systems. The current design incorporates 8 Xilinx 3195 FPGAs, with 3 asynchronous clocks and 5,000 lines of Handel code. However, a commercial design would be simpler, exchanging the flexibility and modularity of the current design with a more cost-effective and robust implementation.

Figure 6 shows the functions of key modules, and how the drive outputs are synthesised. For a first implementation it was decided to mimic in digital hardware the drive synthesis method used in the analogue transmitter. The sensor voltages $\mathrm{V}_{\mathrm{A}}$ and $\mathrm{V}_{\mathrm{B}}$ are sampled by the 18 -bit $\mathrm{AD}-1879$ at $48 \mathrm{kHz}$. The data $\mathrm{D}_{\mathrm{A}}$ and $\mathrm{D}_{\mathrm{B}}$ are sent to the DAC module, which calculates the drive signal $D_{D}$ as a weighted sum (the analogue equivalent is described in section 2.3):

$$
\mathrm{D}_{\mathrm{D}}=\mathrm{D}_{\mathrm{A}}+\mathrm{B}_{\mathrm{S}} \cdot \mathrm{D}_{\mathrm{B}}
$$

where $\mathrm{B}_{\mathrm{S}}$ is the sensor balance, updated each halfcycle, and defined as

$$
\mathrm{B}_{\mathrm{S}}=\frac{\text { amplitude }\left(\mathrm{V}_{\mathrm{A}}\right)}{\operatorname{amplitude}\left(\mathrm{V}_{\mathrm{B}}\right)} \text {. }
$$

$\mathrm{D}_{\mathrm{D}}$ is sent to the AD-1865 to generate the basic drive voltage, $\mathrm{V}_{\mathrm{D} 0}$. The drive gain, $\mathrm{K}_{0}$, defined as

$$
\mathrm{K}_{0}=\frac{\text { drive signal out }(\text { Amps })}{\text { sensor signal in (Volts) }}=\frac{\mathrm{I}_{\mathrm{D}}}{\mathrm{V}_{\mathrm{A}}}
$$

varies greatly under different process conditions. For example, with normal operation a $25 \mathrm{~mm}$ flowtube has $\mathrm{V}_{\mathrm{A}}=0.3 \mathrm{~V}, \mathrm{I}_{\mathrm{D}}=10 \mathrm{~mA}$, and hence $\mathrm{K}_{0}=0.033$. With two-phase flow, values might be $\mathrm{V}_{\mathrm{A}}=0.03 \mathrm{~V}$, $\mathrm{I}_{\mathrm{D}}=100 \mathrm{~mA}$, and $\mathrm{K}_{0}=3.3$, a hundred-fold increase. The need to provide gain with wide range and high precision has resulted in a three step drive waveform synthesis. The AD-1865 provides the basic waveform $\mathrm{V}_{\mathrm{D} 0}$, while Max 532s provide two gain stages. The Max 532 multiplying DAC may be configured in one of two modes: high or low gain. The first stage uses the high gain configuration: the analogue input $\mathrm{V}_{\mathrm{D} 0}$ is multiplied by $4095 / \mathrm{x}$, where $\mathrm{x}$ is the 12 bit gain value sent to the DAC. This stage provides a coarse gain with a high range:

$$
\mathrm{V}_{\mathrm{D} 1}=\frac{4095}{\mathrm{x}} \cdot \mathrm{V}_{\mathrm{D} 0}
$$

The second stage uses the low gain mode. The analogue input $\mathrm{V}_{\mathrm{D} 1}$ is multiplied by $\mathrm{y} / 4095$ where $\mathrm{y}$ is the supplied 12 bit gain value. This fine tunes the first stage to provide a precise overall gain. This is particularly important for high gains (small values of $\mathrm{x}$ ) where the discretisation of the first stage is crude:

$$
\mathrm{V}_{\mathrm{D} 2}=\frac{\mathrm{y}}{4095} \cdot \mathrm{V}_{\mathrm{D} 1}
$$

The terms $\mathrm{B}_{\mathrm{s}}, \mathrm{K}_{0}, \mathrm{x}$ and $\mathrm{y}$ are all calculated by the Power PC. Based on the sensor data $D_{A}$ and $D_{B}$, frequency, phase and amplitude are calculated. From these are derived the sensor balance $\mathrm{B}_{\mathrm{S}}$. A control algorithm to regulate the amplitude of oscillation calculates $\mathrm{K}_{0}$, and then selects $\mathrm{x}$ and $\mathrm{y}$ so that

$$
\frac{\mathrm{y}}{\mathrm{x}} \approx \mathrm{K}_{0}
$$

Beyond the high range and precision afforded by this implementation, two other benefits are provided:

- High update rate. Both the sensor balance and drive gains are updated twice per cycle i.e. at approx. $160 \mathrm{~Hz}$. In the analogue transmitter various filters and delays result in time constants of approx. 2s.

- Negative gain. A digital implementation allows a negative gain to be selected, where the DAC module negates the drive signal to work against the flowtube motion. By contrast, the minimum gain in the analogue transmitter is zero. Negative gain provides a powerful extension to flowtube control, as demonstrated in the next section.

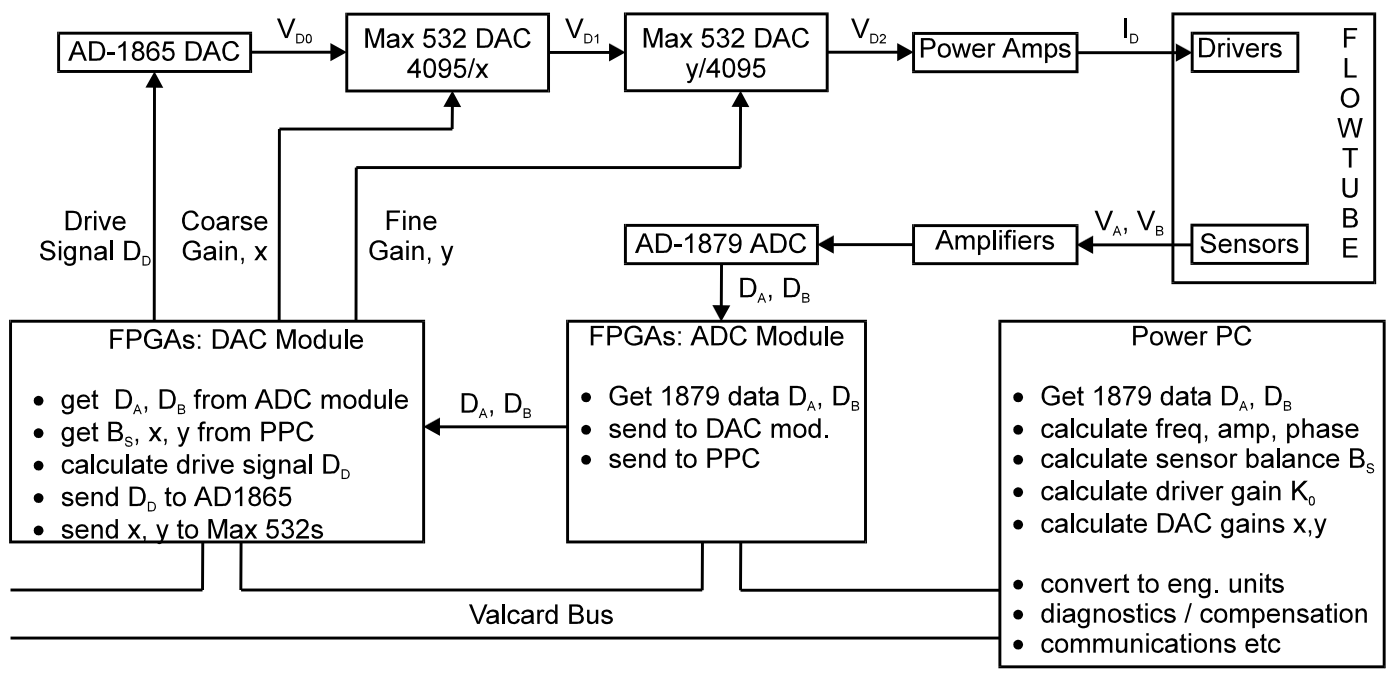

Fig. 6. Subset of digital Coriolis transmitter functionality 


\section{Amplitude control}

The maintenance of flowtube oscillation is the fundamental task of the transmitter; in its absence no flow or density measurement is possible. The primary requirement is the regulation of a steady amplitude during normal operation. However, at start-up, or when modifying the desired amplitude of oscillation (e.g. in response to the presence of an aerated fluid), it is equally important to ensure rapid settling after step changes.

Previous sections have outlined both the analogue transmitter design and the digital hardware implementation of positive (frequency and phase) and negative (amplitude) feedback used to drive the flowtube. Figure 7 shows the corresponding digital control system design. This section provides an overview of the amplitude control algorithm used in the digital transmitter (i.e. C(s)). The background theory is described in detail by Clarke (1998).

Control is exercised by a positive-feedback inner loop whose job is to exactly eliminate damping; the appropriate loop gain is determined by an outer negative feedback loop as shown in Figure 7 . The key step is the use of the natural logarithm of the amplitude, leading to a discrete-time model which is linear for small values of the loop damping (typically $\zeta_{\mathrm{s}}$ ranges from 0.001 to 0.1 for highly aerated flows). This enables:

- A simple design strategy;

- Amplitude-independent transient performance

A further non-linear compensator is added to ensure global linearity and provable stability. In practice this is approximated using a saturating integrator, which retains global stability with only a minor loss in terms of performance.

To ensure the desired amplitude is matched under steady-state conditions, the usual Proportional + Integrator form is the simplest effective controller:

$$
\mathrm{C}(\mathrm{s})=\mathrm{K}_{\mathrm{p}}\left(1+1 / \mathrm{sT}_{\mathrm{i}}\right) \text {, }
$$

with gain $K_{p}$ and integral time $T_{i}$. For this type of system with two integrator poles and a zero, overshooting in response to step demands can be prevented if the reference signal $\mathrm{a}_{0}$ is passed through a simple first-order filter - the discretisation of $1 /(1+$ $\mathrm{sT}_{\mathrm{i}}$ ) - in order to provide a "pseudo-setpoint”.

The control algorithm is carried out on the Power PC every half-cycle (typically at $160 \mathrm{~Hz}$ ) based on the amplitude calculations discussed in section 6. The desired gain $\mathrm{K}_{0}$ is mapped onto settings for $\mathrm{x}$ and $\mathrm{y}$ for the Max-532 s, and sent out via the Valcard bus.

The effectiveness of the control design is shown in Figure 8, which compares the start-up behaviour of

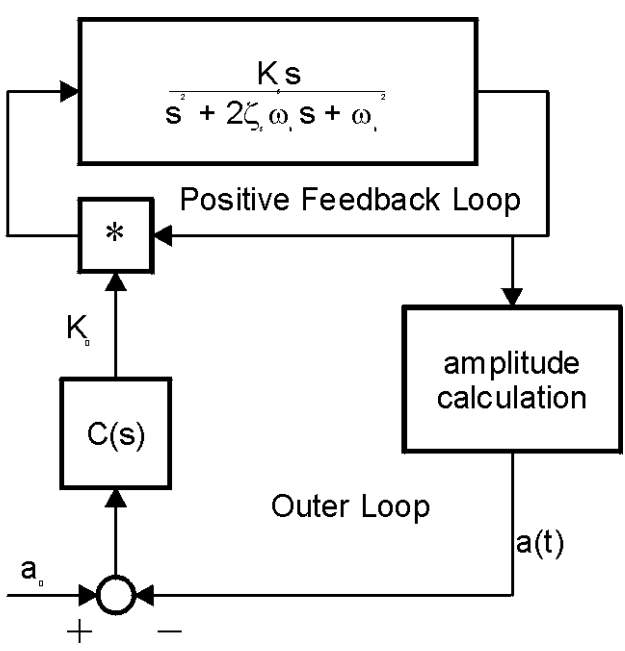

Fig. 7. Control design for amplitude regulation

the analogue and digital transmitters, both driving a $25 \mathrm{~mm}$ flowtube under low flow conditions. The nonmonotonic behaviour of the analogue transmitter is caused by the deployment of a fixed, maximum drive gain on start-up. The analogue feedback system is switched on when the desired setpoint is approached, at about $\mathrm{t}=3 \mathrm{~s}$. Note also the exponential rate of increase, limited by the maximum drive gain, and the slight overshoot, so that the set point is finally settled on at about $\mathrm{t}=11 \mathrm{~s}$. By contrast the digital controller, which has effectively infinite drive gain available, provides an initially linear rise in amplitude (limited only by the maximum available drive current) with no overshoot. It settles at the setpoint in less than $1 \mathrm{~s}$.

Once the setpoint has been reached, disturbance rejection is also excellent. In 'quiescent' conditions (in the absence of flow or external vibration), amplitude variations of less than 20 parts per million per cycle have been recorded. More details are given in the next section which discusses measurement performance. As discussed later, measurement precision is strongly correlated to amplitude stability, and hence there is a feedback relation between the quality of measurement and control for the flowmeter.

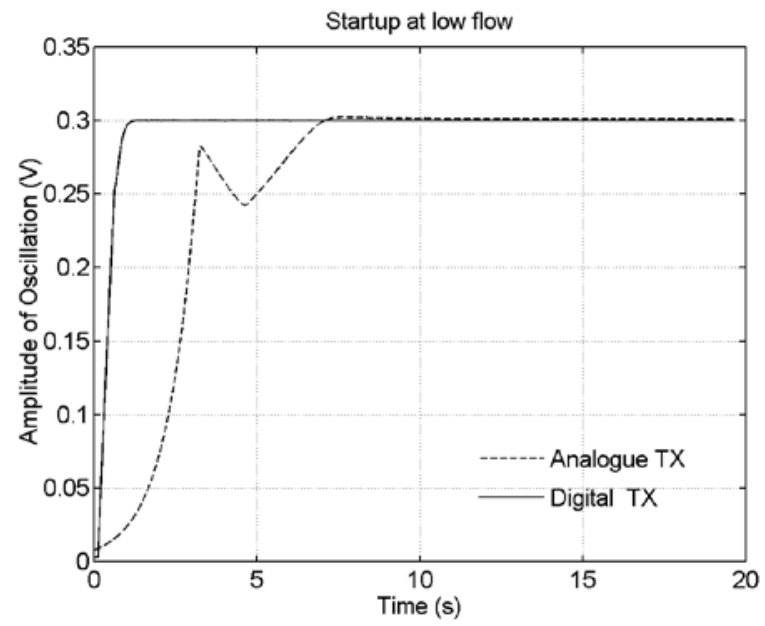

Fig. 8. Start-up for analogue and digital transmitters 

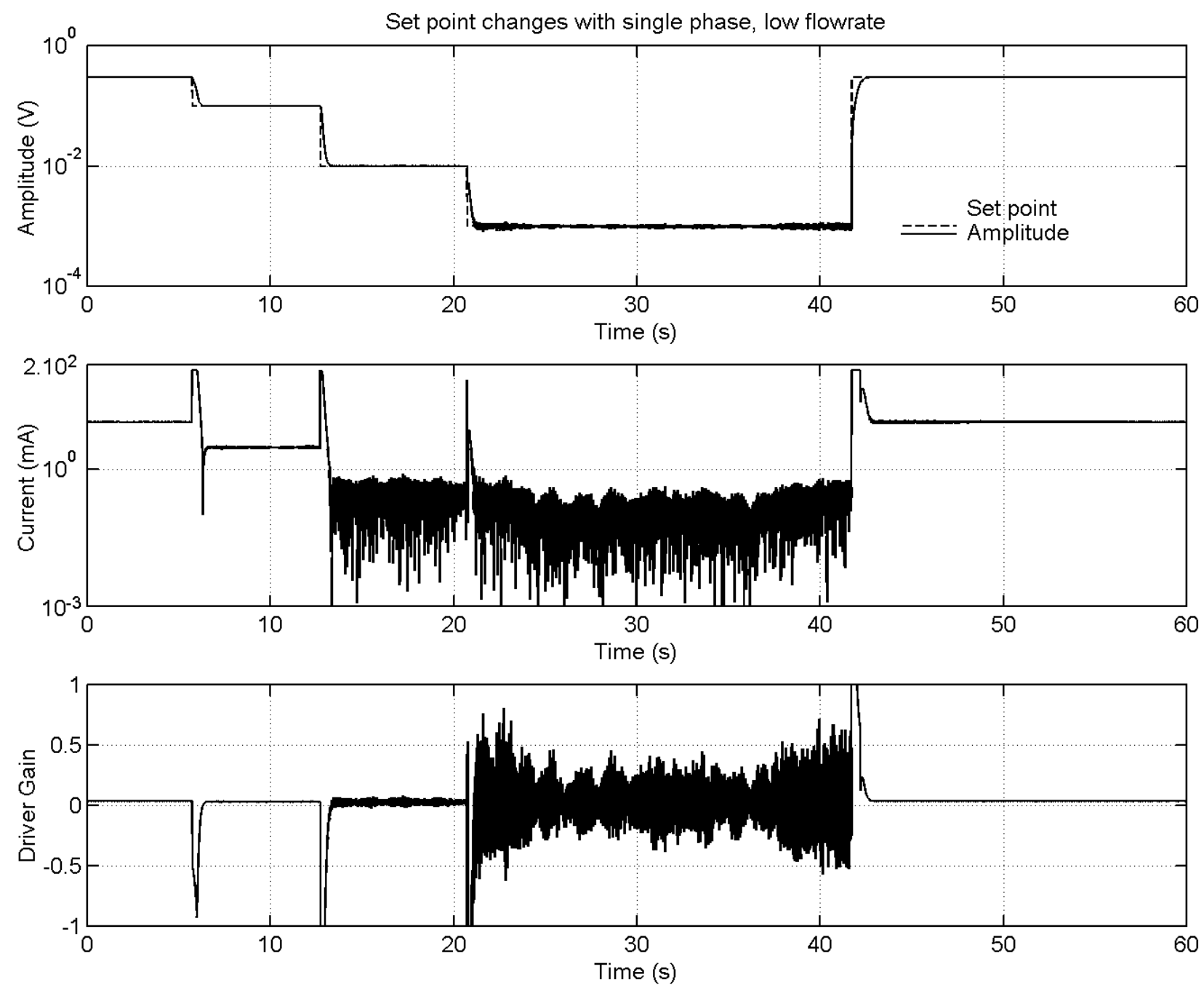

Fig. 9. Performance of control algorithm with set point varying from $0.3 \mathrm{~V}$ down to $0.001 \mathrm{~V}$

The interaction between amplitude, current and drive gain is illustrated in Figure 9, which demonstrates the performance of the control algorithm over two and a half orders of magnitude in the amplitude of oscillation. The top graph shows the set point and amplitude of oscillation as the set point is changed manually from $0.3 \mathrm{~V}$ to $0.1 \mathrm{~V}, 0.01 \mathrm{~V}$ and $0.001 \mathrm{~V}$ (equivalent to $5 \mu \mathrm{m}$ physical vibration) respectively, before returning to $0.3 \mathrm{~V}$. Each set point change is matched rapidly by the controller with no discernible overshoot. Note that the transients look the same even though the scales are logarithmic, reflecting the use of the logarithm of the amplitude. At $0.001 \mathrm{~V}$, there is a much higher level of noise, but this can be attributed to a number of factors, including the loss of 8.2 bits of ADC precision as well as the relative increase in mechanical noise.

The middle graph shows the magnitude of the drive current reducing with amplitude from $11.2 \mathrm{~mA}$ (at $0.3 \mathrm{~V}$ ) down to $0.13 \mathrm{~mA}$ (at $0.001 \mathrm{~V}$ ). During set point changes, the maximum available current $(150 \mathrm{~mA})$ is used. Note that the sign of the current is indicated by the drive gain, shown in the lowest graph. Thus set point reductions result in maximum current with negative gain to oppose the flowtube vibration. If the absolute drive current is considered, then the average required for oscillation at $0.001 \mathrm{~V}$ is only $0.017 \mathrm{~mA}$. Thus, low-amplitude operation under normal flow regimes requires very low drive power, suggesting a two-wire design might indeed be practical.

The drive gain graph shows the utility of negative gain, for reducing the amplitude of vibration and for maintaining control at low amplitudes. Note that the $y$-axis is truncated; the maximum value of the gain when the set point is restored to $0.3 \mathrm{~V}$ (at about 42s) is about 150 , demonstrating the range of gain values that may be required during flowtube operation. This is further illustrated in the next example.

For Figure 9, the set point was changed manually. Figure 10 shows how the set point is selected automatically in responses to changes in damping. At a high flow rate, increasing levels of air were injected into the flow stream, as shown by the apparent density. As discussed section 8, this is not the true air/water mixture density, but it does give an indication of the relative level of aeration. The dynamics of gas/liquid flow produces mechanical energy losses, resulting in increased damping. This is reflected in the drive gain, which increases from 

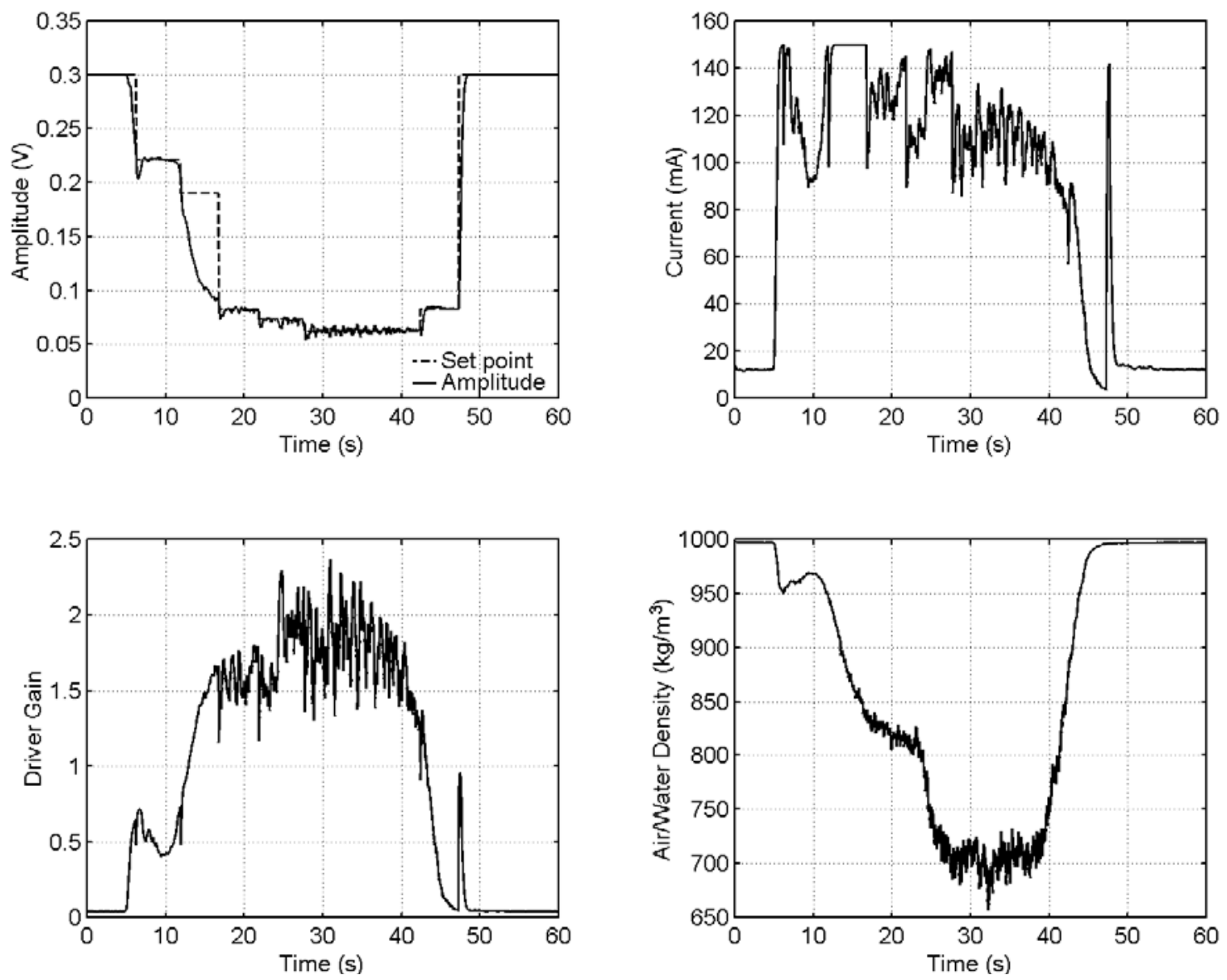

Fig. 10. Automatic selection of amplitude set point in response to variable damping from two-phase flow

0.034 with no air up to over 2.0 with high aeration. The digital transmitter can deliver the required gain, but there is also a drive current limit of $150 \mathrm{~mA}$. For example during the interval $t=13-17 \mathrm{~s}$, the drive current saturates, and as damping continues to increase the amplitude of oscillation drops. In effect the set point is no longer achievable. An algorithm has been deployed which adjusts the set point to ensure sustainability. As can be seen, several set point changes occur, ensuring that the amplitude is actively controlled. To prevent excessive jitter, there is a minimum time between set point changes; this explains the delay from $\mathrm{t}=13-17 \mathrm{~s}$ before a new sustainable set point is selected.

The desirability of ensuring a stable amplitude of oscillation is further discussed in the next section, which describes the measurement algorithms employed in the digital transmitter.

\section{Measurement Algorithms}

The essential measurement requirements are the calculation of the frequency, amplitude, and phase difference (here called the key parameters) between the sensor signals. The mapping to engineering units also requires the flowtube temperature to compensate for variable tube stiffness. Thus, the mass flow measurement is is derived from the phase difference $\Phi$ using an equation of the form:

$$
\mathbf{r} \boldsymbol{\alpha}=\left(\mathrm{FC}_{1} \cdot \Delta \mathrm{T}+\mathrm{FC}_{2}+\mathrm{FC}_{3} \cdot \Delta \mathrm{T}^{2}\right) \cdot \frac{\tan (\Phi)}{\mathrm{f}},
$$

where:

$\mathrm{FC}_{\mathrm{i}}$ are the flow calibration coefficients;

$\Delta \mathrm{T}$ is the deviation from the calibration temperature; $\Phi$ is the phase difference;

$\mathrm{f}$ is the resonant frequency.

Note that in radians, $\Phi$ is close to zero (typically no greater than 0.07), so that the difference between $\Phi$ and $\tan \Phi$ is very small (less than $0.2 \%$ ); it is not uncommon for the tangent function to be neglected.

Similarly, the density measurement $\rho$ is derived from the resonant frequency using

$$
\rho=\frac{\left(\mathrm{DC}_{1} \cdot \Delta \mathrm{T}+\mathrm{DC}_{2}\right)}{\mathrm{f}^{2}}+\mathrm{DC}_{3}+\mathrm{DC}_{4} \cdot \Delta \mathrm{T},
$$

where:

$\mathrm{DC}_{\mathrm{i}}$ are the density calibration coefficients; $\Delta \mathrm{T}$ is the deviation from the calibration temperature; $\mathrm{f}$ is the resonant frequency.

There appears at this stage little scope for using a digital transmitter to improve the conversion to engineering units. Once the key parameters have been determined the remaining calculations are essentially mundane and not considered further. 
As this paper provides an outline of all aspects of the digital Coriolis flowmeter, only an overview is provided of the key calculations. A detailed description of the measurement and compensation algorithms will be the subject of a future paper.

Assuming that the raw data are sampled at high speed and computing power is plentiful, there are numerous ways of extracting the key parameters, and several strategies have been implemented and compared. These include digital equivalents of analogue transmitter methods such as synchronous demodulation (Bergovic et al., 1993) and time delay. Time delay, the most widely used technique, employs the time between zero crossings on the sensor signals to measure phase difference. Given zero crossing times $t_{A}$ and $t_{B}$ on sensors $A$ and $B$ respectively, the phase difference $\Phi$ is given by:

$$
\Phi=\left(\mathrm{t}_{\mathrm{A}}-\mathrm{t}_{\mathrm{B}}\right) \cdot 2 \pi \mathrm{f} .
$$

Other algorithms are only viable in a digital implementation, such as Fourier techniques or adaptive least squares. A Fourier-based technique, described in the following subsection, has been found to be very precise, and is the basis for all the results described in the remainder of this paper.

\subsection{Fourier Calculation}

As illustrated in Figure 11, data from each sensor is processed in batches consisting of whole cycles centred on successive zero crossings, $\mathrm{k}=1,2,3, \ldots$, occurring at time $\mathrm{t}(\mathrm{k})$. There is thus a $180^{\circ}$ overlap between consecutive 'cycles'. With a phase difference of up to $4^{\circ}$, the cycles in data streams $D_{A}$ and $D_{\mathrm{B}}$ (Figure 6) do not coincide exactly but are easily paired. The frequency of cycle $k$ on sensor $\mathrm{X}$ (= A or $\mathrm{B})$ is given by

$$
f_{X}(k)=\frac{1}{t_{X}(k+1)-t_{X}(k-1)}
$$

The zero crossings are located using a curve fitting technique. Additional algorithms are used to monitor and eliminate DC offset and gain mismatch between positive and negative voltages (caused by differential front-end circuitry).

The amplitude $a_{x}$ and 'phase' $\phi_{x}$ of each cycle are calculated using standard Fourier integrals:

$$
\begin{gathered}
I_{X C}=\int_{t_{X}(k-1)}^{t_{X}(k+1)} D_{X}(t) \cdot \cos \left[2 \pi f_{X}(k) \cdot t^{\prime}\right] d t, \\
I_{X S}=\int_{t_{X}(k-1)}^{t_{X}(k+1)} D_{X}(t) \cdot \sin \left[2 \pi f_{X}(k) \cdot t^{\prime}\right] d t, \\
a_{X}(k)=\sqrt{I_{X S}^{2}+I_{X C}^{2}}, \phi_{X}(k)=\tan ^{-1} \frac{I_{X C}}{I_{X S}},
\end{gathered}
$$

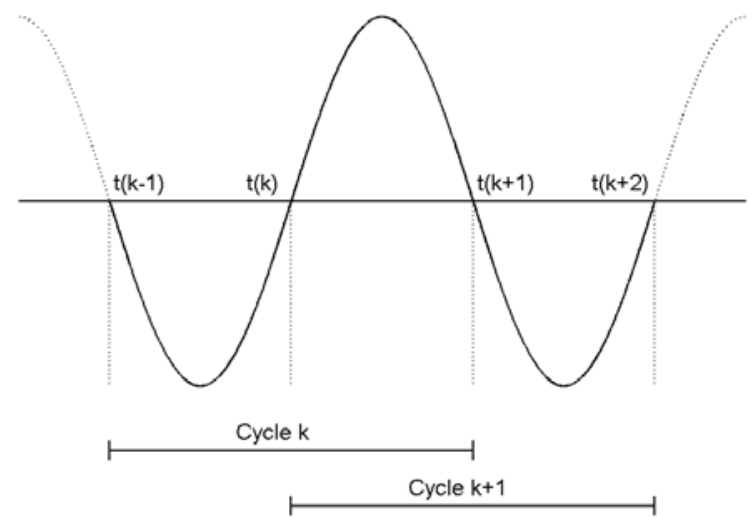

Fig. 11. Data batches or 'cycles' used to process sensor data

where $\mathrm{t}^{\prime}$ starts from zero i.e. $\mathrm{t}^{\prime}=\mathrm{t}-\mathrm{tx}_{\mathrm{x}}(\mathrm{k}-1)$. The 'phase' $\phi_{\mathrm{x}}$ is interpreted in the context of a single waveform as being the offset between the start of the cycle (i.e., the zero-crossing point) and the point of zero phase for the component of $\mathrm{D}_{\mathrm{X}}$ of frequency $f_{X}(k)$. Since this phase is an average over the full cycle, it may equally be considered an offset from the midpoint of the cycle. For measurement purposes, the average of the two sensor frequencies for each cycle, $f_{A V}$ is used as the 'frequency of oscillation'. The phase difference is calculated from the sensor phase offsets relative to the respective cycle midpoints; a simple formulation is:

$$
\Phi(\mathrm{k})=\left[\frac{\phi_{\mathrm{A}}(\mathrm{k})}{\mathrm{f}_{\mathrm{A}}(\mathrm{k})}-\frac{\phi_{\mathrm{B}}(\mathrm{k})}{\mathrm{f}_{\mathrm{B}}(\mathrm{k})}+2 \pi\left(\mathrm{t}_{\mathrm{A}}-\mathrm{t}_{\mathrm{B}}\right)\right] \mathrm{f}_{\mathrm{AV}}(\mathrm{k})
$$

Here the term $2 \pi\left(t_{A}-t_{B}\right)$ is the phase offset due to the different start times for the two data sets. Eq. (16) demonstrates how, in this formulation, the Fourier technique can be seen as a refinement of the time delay technique given in Eq. (11), in which only the phase of the resonant frequency is considered. Other, more elaborate, algorithms may be preferred which compare the phases of simultaneous data sets from sensors $\mathrm{A}$ and $\mathrm{B}$.

\subsection{Repeatability}

Repeatability results for a $25 \mathrm{~mm}$ flowtube operating in a variety of conditions are shown in Table 1 . Given a fixed measurement input, standard deviations were calculated from 10,000 cycles. Absolute, not percentage, values are shown due to the use of zero phase input in several table rows.

Table 1. Results of Fourier-based calculations, with frequency $82-95 \mathrm{~Hz}$, phase $0-4^{\circ}$, amplitude $0.3 \mathrm{~V}$

\begin{tabular}{clll}
\hline & \multicolumn{3}{c}{ Standard Deviation } \\
& Freq. (Hz) & Phase $\left(^{\circ}\right)$ & Amp. (V) \\
\hline Sig. Gen. & $9.94 \mathrm{e}-4$ & $1.52 \mathrm{e}-3$ & $5.89 \mathrm{e}-6$ \\
Empty tube & $3.20 \mathrm{e}-4$ & $1.31 \mathrm{e}-3$ & $6.10 \mathrm{e}-6$ \\
0\% flow & $4.87 \mathrm{e}-4$ & $6.71 \mathrm{e}-3$ & $2.31 \mathrm{e}-5$ \\
$10 \%$ flow & $2.32 \mathrm{e}-3$ & $1.41 \mathrm{e}-2$ & $4.94 \mathrm{e}-4$ \\
$70 \%$ flow & $7.96 \mathrm{e}-3$ & $1.06 \mathrm{e}-1$ & $3.87 \mathrm{e}-3$ \\
\hline
\end{tabular}


Perhaps the most surprising result is that an empty flowtube is capable of generating a signal with more stable frequency and phase characteristics than a modern digital signal generator.

The measurement variations seen in Table 1 are of course a function of the process and flowtube as well as the digital transmitter, thus increased phase variation might be expected with non-zero flow. Less expected, however, is the increasing noise of frequency with increased flow. The experiments with $0 \%, 10 \%$, and $70 \%$ flow rate were all carried out with a full flowtube, and so assuming a constant density of water the increased frequency noise must be due to another source. The corresponding increase in amplitude variation is an important factor, as discussed shortly. However, the primary conclusion drawn from experimental data such as the results in Table 1 is that the resolution of the transmitter itself is unlikely to be a significant source of precision error, particularly in industrial applications.

Figure 12 shows how the repeatability of the key parameters is reflected in the massflow measurement. The total flow from two digital flowmeters $(25 \mathrm{~mm}$ and $50 \mathrm{~mm}$ ) were compared over 20 consecutive flow runs at $1 \mathrm{~kg} / \mathrm{s}$. The distribution of the difference between the totals was found to be normal, with a standard deviation of $0.0057 \%$. Note that this is a far higher level of repeatability than that of a conventional flow laboratory, and hence the need to cross compare the totals from two meters.

\subsection{Accuracy}

Figure 13 shows, for the digital transmitter working with a $25 \mathrm{~mm}$ flowtube, the mass-flow error (obtained from batch trials) over the range of the instrument, which has a turndown of 100:1. Three measurements are provided at each flow rate to give a further indication of repeatability (which here includes the repeatability of the flow rig). The upper and lower bounds show the (static) uncertainty claimed for the commercial flow meter. Clearly in this case the digital transmitter performs well within this

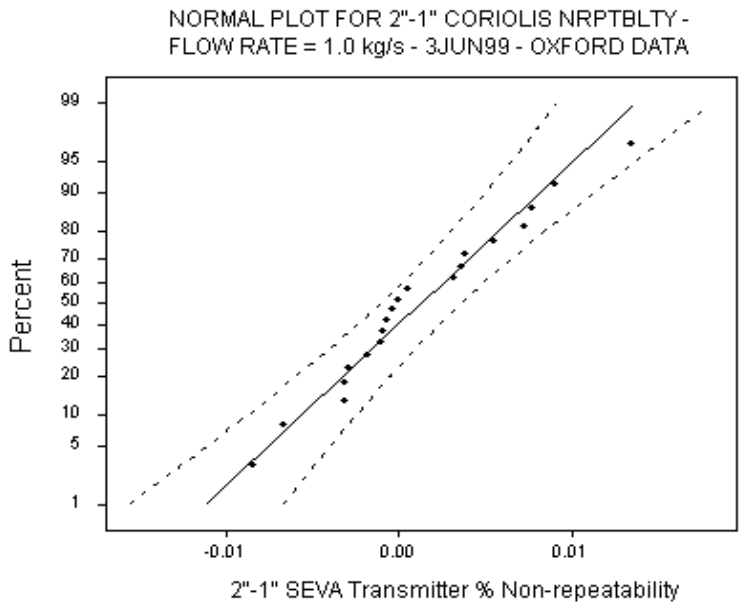

Fig. 12. Flow repeatability comparing two meters over 20 consecutive trials; s.d. $=0.0057 \%$

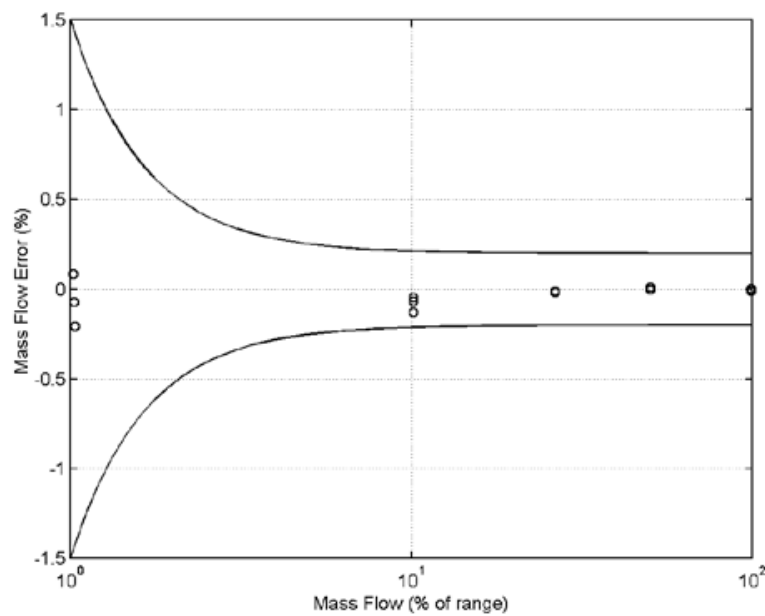

Fig. 13. Absolute accuracy of digital transmitter

specification. In practice it has been found that the linearity of the reading over the flow range depends upon the particular flowtube. Again, the most important conclusion is that the digital transmitter is not a significant source of error. Indeed its high precision might be used to provide an additional correction for any non-linearities observed during flowtube characterisation.

\subsection{Dynamic effects}

Early experimental evidence (e.g. Table 1) suggested a relationship between the stability of the amplitude of oscillation and the cycle-to-cycle repeatability of the phase and frequency measurements. Indeed, the provision of very precise amplitude control (to improve measurement repeatability) was the motivation for the three stages of drive waveform synthesis (Section 4). Further work has demonstrated that the interaction between amplitude and measurement stability can be established, enabling compensation for amplitude dynamics.

Figure 13 illustrates the relationship between the observed frequency of oscillation and changes in the amplitude of oscillation. The data was obtained from a $25 \mathrm{~mm}$ flowtube filled with water and sealed at each end (to ensure zero flow). It was driven by the digital transmitter which generated a random amplitude set point. The top graph shows the amplitude variations, while the next graph shows the corresponding observed frequency of oscillation, both calculated every half-cycle (i.e. at about $160 \mathrm{~Hz}$ ). From Table 1, a full flowtube with zero flow and stable amplitude has a typical standard deviation of $4.9 \mathrm{e}-4 \mathrm{~Hz}$ for frequency. In Figure 13, the s.d. of the observed frequency is $5.4 \mathrm{e}-2 \mathrm{~Hz}$, some 100 times greater. With a fixed process density, no flow, and no external vibration, the frequency deviations must be attributable to the variations in amplitude.

The third graph displays $-r_{a}$, where $r_{a}$ is the relative rate of change of amplitude on sensor $\mathrm{A}$, using:

$$
r_{a}(k)=\frac{a(k+1)-a(k-1)}{a(k)} . f(k) \times 100 \%,
$$



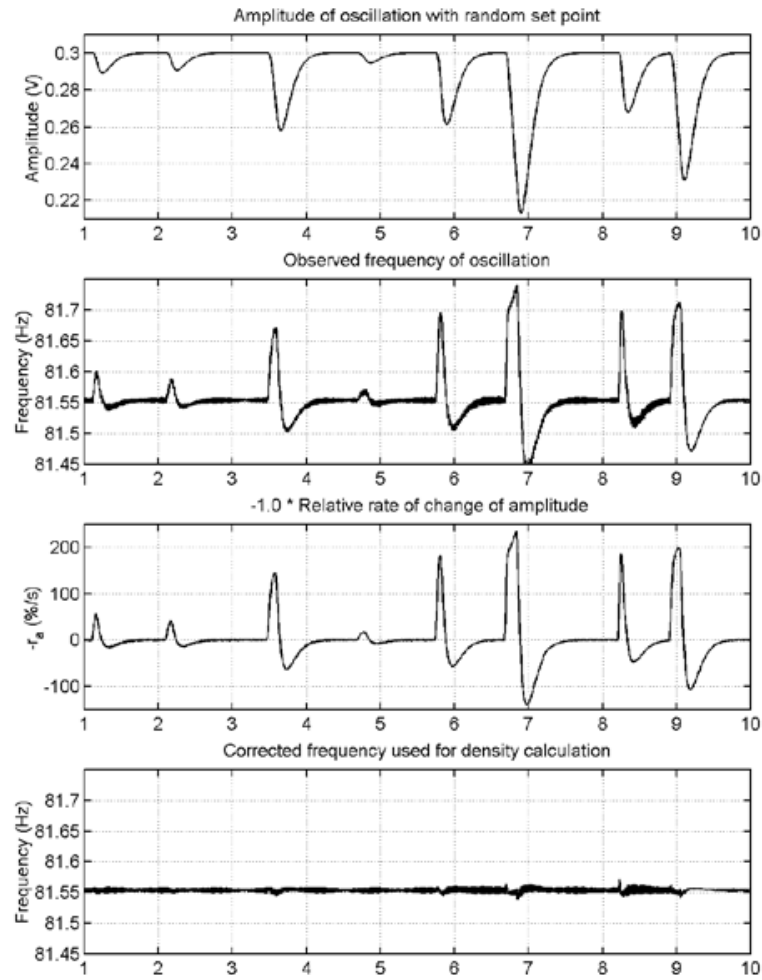

Fig. 14. Relationship between amplitude change and observed frequency of oscillation

where $a(k)$ is the amplitude of oscillation and $f(k)$ is the observed frequency over the cycle centred on zero-crossing $k$, all on Sensor A. The form of this equation is derived as follows. Assuming a linear variation of amplitude over each cycle, the average amplitude over a cycle (as calculated by the Fourier technique) is equal to the instantaneous value at the midpoint of the cycle. However, referring back to Figure 11 which shows the overlapping cycles used in measurement calculation, the midpoint of cycle $\mathrm{k}$ is the start-point of cycle $\mathrm{k}+1$ and the end-point of cycle $\mathrm{k}-1$. It thus follows that the relative rate of change of amplitude over cycle $\mathrm{k}$ takes the form of Eq. 17.

The high correlation between the middle graphs of Figure 14 (i.e. between the observed frequency of oscillation and $-r_{a}$ ) is readily seen. An algorithm has been implemented that applies a correction to the observed frequency based on $r_{a}$. The lowest graph shows the corrected frequency, which has a smaller variance (the s.d. is $6.9 \mathrm{e}-3 \mathrm{~Hz}$, a reduction by a factor of 8.4). This corrected frequency is used to calculate density, thus reducing measurement noise caused by amplitude variation.

Of course, for Figure 14, a random set-point has been used to generate large amplitude swings. Under normal (single phase) operating conditions with a fixed set-point, the correction leads to a s.d. reduction in frequency of only perhaps $20 \%$.

The impact of $r_{a}$ on the phase measurement is typically greater, and results suggest that correcting for $r_{a}$ is essential to realise the full precision of the digital transmitter. This is illustrated in Figure 15, which shows phase data calculated simultaneously by three methods, from a $25 \mathrm{~mm}$ flowtube filled with water at a low flow rate. The outer band of data is calculated using the Fourier method, while the middle band results from the time delay method. Before the dynamic effects were discovered, it was a source of considerable frustration that the variance of phase data from the Fourier technique (using large quantities of high precision data and much computing power) was consistently about twice that from the time delay technique, which simply observes the time between zero crossings.

An explanation for the higher precision of the time delay method is that it makes fewer assumptions. Specifically, Fourier methods assume a stationary waveform, which implies in this context a constant amplitude (and indeed frequency and phase difference) over each cycle. This can only be an approximation in real, continuously varying data.

The lower graph in Figure 15 shows the corresponding behaviour of $r_{a}$, and visual inspection again suggests a strong correlation between $r_{a}$ and both the Fourier and time-delay phase data. Note that large values of $r_{a}$ (up to $10 \%$ per second) are generated, even though the amplitude itself is stable due to the action of the control algorithm. This is explained by considering the high update rate: from Table 1, a typical amplitude standard deviation at low flow is $4.9 \mathrm{e}-4 \mathrm{~V}$ or $0.16 \%$ of reading, but this is achieved by running the control algorithm at $160 \mathrm{~Hz}$.
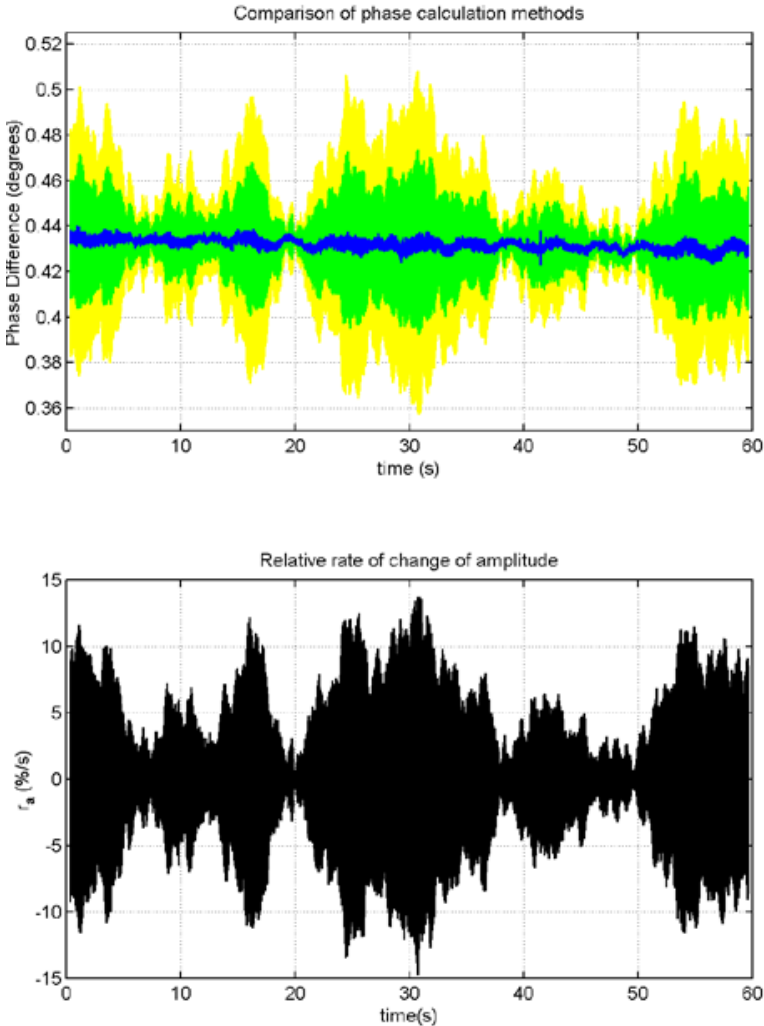

Fig. 15. (above) phase calculated by three methods; (below) corresponding value of $r_{a}$ 
The inner-most band on the upper graph in Figure 15 shows the same Fourier phase data after a correction has been applied, based on $r_{a}$. It is clear that the s.d. has been reduced, by a factor of 11.6 over the Fourier data and 6.1 over the time delay data. Given that all three data sets were calculated simultaneously and hence using the same 'true' process flow, it is reasonable to assume that both uncorrected data sets are contaminated with errors associated with $r_{a}$. In the corrected data, there is no longer a marked correlation with $r_{a}$, instead other trends become apparent. The 3s cycling in the data is due to the poor action of the variable speed driver of the rig pump, leading to low frequency flow pulsation (Cheesewright and Clark, 1998). Thus the data appears clean enough to allow condition monitoring of nearby plant components.

The frequency and phase errors associated with $r_{a}$ have no impact on the accuracy of the flow meter over any significant period of time, as long as on average the amplitude is constant. They are however highly influential on the dynamic response to flow step changes, and in minimising the effect of external vibration, as illustrated in Figure 16, which shows the same three calculation methods as Figure 15. In the upper graph a number of small step changes in flow take place. These are clearly visible in the corrected data, but are masked by the effects of $r_{a}$ in the raw data. In the lower graph, the action of an actuator adjacent to the flowtube causes a 'ringdown' effect on the raw data, starting at $\mathrm{t}=20 \mathrm{~s}$ and $\mathrm{t}=40 \mathrm{~s}$. In the corrected data ring-down is substantially reduced.

The corrections to frequency and phase have been implemented in several ways, including simple equations with empirically-derived coefficients, and using system identification techniques. The most robust approach entails calculating a separate estimate of $r_{a}$ for each sensor waveform (A and B), and applying separate corrections to the individual phases $\phi_{\mathrm{A}}$ and $\phi_{\mathrm{B}}$ (Eqs. 13-16). This results in s.d. reduction (over uncorrected Fourier) by a factor of 520 in all observed conditions and on all flowtube sizes without further tuning or identification. Clearly such a correction can only be applied to a Fourier based calculation with separate phase estimates for sensors $\mathrm{A}$ and $\mathrm{B}$, and in particular is not applicable to the time delay technique (Eq. 11), although some of the less effective and robust methods are suitable.

The description of these dynamic effects has necessarily been superficial in order to transmit the fundamental ideas concisely. They have been presented here from an empirical perspective, reflecting their discovery. The mechanisms involved are not as yet completely understood, though they are thought to include the following:

- A change in physical amplitude causes a change in the apparent frequency (and hence also 'phase') of the output of a velocity sensor.

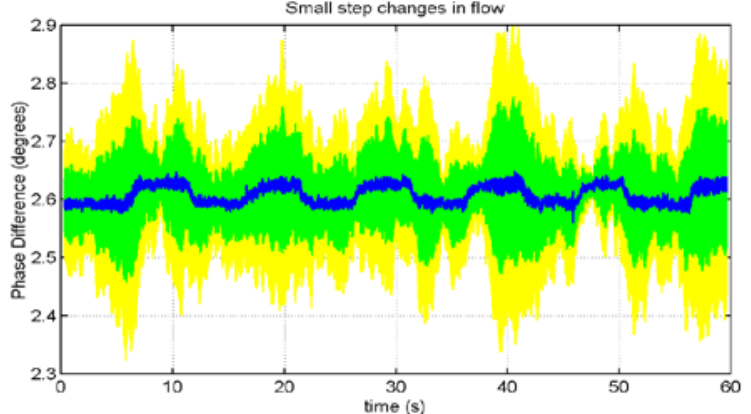

Medium flow, external vibration

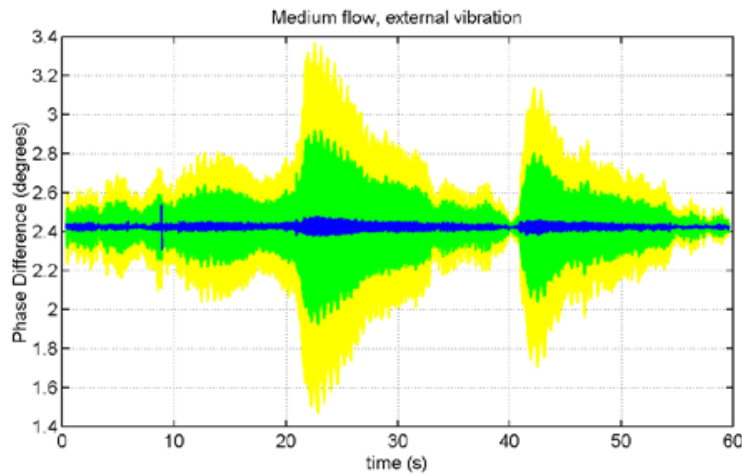

Fig. 16. phase calculated by three methods: (above) for flow step changes; (below) with external vibration

- The sensors are of course physically coupled by the flowtube, so that the dynamics of the sensor signals are related.

- The feedback relation between the control action and the amplitude calculation is clearly highly influential. When these effects are better understood, a new control strategy might be developed to optimise phase and frequency precision instead of amplitude stability.

Clearly for this flowtube design, and perhaps for others (using velocity sensors), the influence of $r_{a}$ on frequency and phase is a constraint on measurement precision, unless compensation is applied. It has been shown that without compensation Fourier techniques perform worse than the simple time-delay technique. Indeed, the use of higher precision or faster digital technology (e.g. ADCs) would not improve the precision of the Fourier or time delay algorithms (or presumably other methods). However if dynamic compensation is applied, improved precision may be possible. After all, it has been shown that a correction based on the instantaneous rate of change (an inherently noisy calculation) - Eq. 17 - causes a substantial reduction in measurement noise.

It is believed that further improvements are possible once the mechanisms of these dynamic effects are better understood. Thus far, corrections have only been made for dynamic changes in amplitude, but clearly the dynamics of phase and frequency must also have an impact upon the observed measurements. The key requirement is to develop a dynamic model of the flowtube to explain and predict these observed deviations in measurement. 


\section{Batching from empty}

The last few sections provide implementation details of particular aspects of the digital transmitter. This section demonstrates how these features combine to improve the performance of the transmitter in dealing with a difficult application condition.

There are many processes, particularly in the food and petrochemical industries, where the high accuracy and direct mass-flow measurement provided by Coriolis technology would be beneficial in the metering of batches of material. In many cases, however, it is not practical (or highly inefficient) to ensure that the flowmeter remains full of fluid from start to end of the batch. For example, in filling or emptying a tanker, air entrainment is difficult to avoid. In food processing, hygiene regulations may require the cleaning out of pipework between batches. However, large errors are induced in conventional Coriolis meters when 'wet and empty'. Hydraulic shock and a high drive gain requirement may be caused by the onset of flow in an empty flowtube, leading to large measurement errors and stalling. Most Coriolis manufacturers are thus unable to recommend the use of their products in batch applications unless the flowtube can be kept full.

The digital transmitter has been designed to be robust to the conditions experienced when batching from empty. Specifically:

- The amplitude controller has a rapid response.

- The high gain range prevents flowtube stalling.

- Measurement data can be calculated down to $0.1 \%$ of the normal amplitude of oscillation.

- There is compensation for the rate of change of amplitude.

This is illustrated in Figure 17, which shows the response of the digital transmitter driving a wet and empty $25 \mathrm{~mm}$ flowtube during the first seconds of the onset of full flow. Note that prior to the onset of flow (at about $0.4 \mathrm{~s}$ ), the drive gain required to drive the wet and empty tube is higher (approx. 0.1) than that needed for a full flowtube (0.034). The onset of flow is characterised by a substantial increase in gain and corresponding drop in amplitude of oscillation. At about $1 \mathrm{~s}$ the selection of a reduced setpoint assists in the stabilisation of amplitude while the full flow regime becomes established. After about 2.75s the last of the entrained air is purged, the conventional setpoint is restored and the drive gain assumes the nominal value of 0.034 . The raw and corrected phase difference behaviour is shown in the lowest graph (using the same format as Figures 14 and 15).

The first observation is that phase data is given continuously throughout the transition. In similar circumstances the analogue transmitter stalls, and is unable to provide measurement data until the required drive gain returns to a near-nominal value
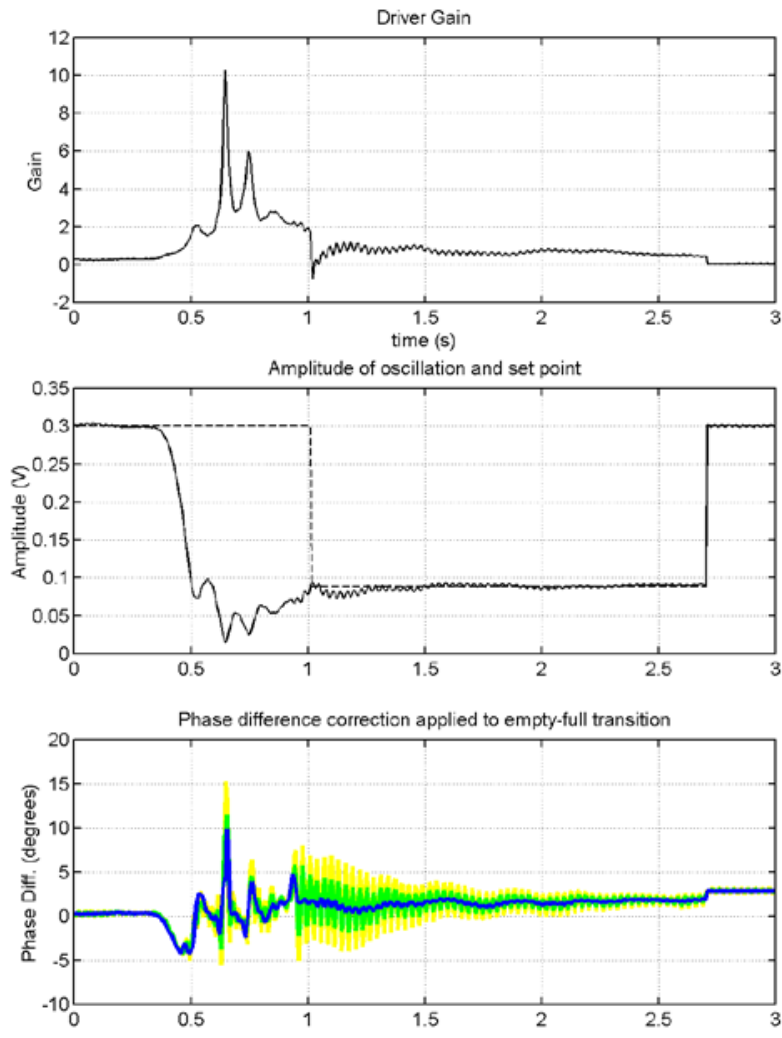

Fig. 17. Response of digital transmitter to the onset of flow onto a 'wet and empty' $25 \mathrm{~mm}$ flowtube

and the (lengthy - Figure 8) start-up procedure is completed. Secondly, the correction for the rate of change of amplitude is clearly beneficial, particularly after 1s. Oscillations in amplitude cause substantial swings in the Fourier-based and time-based calculations of phase, but these are substantially reduced in the corrected phase measurement. Even in the most difficult part of the transition, 0.4-1s, the correction provides some noise reduction.

Of course there are still erroneous data in this interval: flow generating a phase difference in excess of about 5 degrees is physically not possible. However, from a SEVA perspective (Henry and Clarke, 1993), this phase measurement still constitutes 'raw data'. A higher level validation process might well identify the data from $0.4-1$ s as unrepresentative of the true process value (based on the gain, amplitude and other internal parameters), and generate a 'Dazzled' mass-flow status, thus suppressing extreme measurement values.

Having examined in some detail the response of the digital transmitter to the onset of flow, experimental data is presented which demonstrates the resulting improvements in accuracy and repeatability. Figure 18 shows the experimental equipment. Fluid is pumped through magnetic and Coriolis flowmeters into a weighing tank. Valves are used to ensure that the magnetic flowmeter is always full, while the Coriolis flowtube begins each batch empty. At the start of the batch the totalisers in the magnetic and Coriolis flowmeters are reset and flow commences. At the end of the batch the shutoff valve is closed 


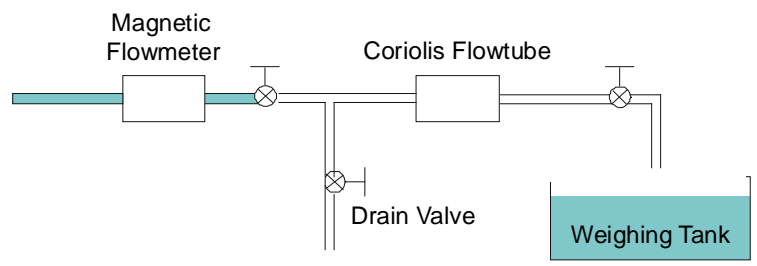

Fig. 18. Experimental equipment for batching from empty trials in industrial flowlab

and the totals are frozen (hence the Coriolis meter is full at the end of the batch). Three totals are recorded, from the magnetic and Coriolis flowmeters, and from the weighscale. These totals are not expected to agree, as there is a finite time delay before the Coriolis meter and then finally the weighing tank observes fluid flow. Thus, it would be expected that the magnetic flowmeter would record the highest total flow, then the Coriolis meter, with the weighing tank observing the lowest total.

Figure 19 shows the results obtained from a series of trial runs, each of about $550 \mathrm{~kg}$. The data shown is the offset observed between the weighscale and either the magnetic flowmeter or the Coriolis meter. As explained above, positive offsets are expected from both instruments. The (always full) magnetic flowmeter delivers a consistently positive offset, with a repeatability (defined here as the maximum difference in reported value for identical experiments) of $4.0 \mathrm{~kg}$. The analogue transmitter generates large negative offsets, with a mean of $-164.2 \mathrm{~kg}$ and a repeatability of $87.7 \mathrm{~kg}$. This poor performance is attributable to its inability to deal with the onset of flow and the varying time taken to restart the flowtube. By contrast the digital transmitter shows a positive offset averaging $25.6 \mathrm{~kg}$ and a repeatability of $0.6 \mathrm{~kg}$.

It would be difficult to assess the 'true' mass-flow through the flowtube, given its initially empty state. The reported total mass falls between that of the magnetic flowmeter and the weighscale, as expected. It is probably impractical to pursue the issue of accuracy further, and in an industrial application the issue of repeatability is often of greater importance (batch 'recipes' are often adjusted to accommodate offsets). Of course, the repeatability of the filling process (which here seems surprisingly good) is a lower bound on the repeatability of the Coriolis meter total. Thus it cannot be guaranteed that similar repeatability could be achieved in an arbitrary industrial batch process. However, it is clear that the digital transmitter provides a substantial performance improvement over its analogue equivalent under the same conditions. Again, the conclusion drawn is that the digital transmitter in these conditions is not a significant source of measurement error.

\section{Two-phase flow}

Another industrially-important condition which presents difficulties for analogue transmitters is sporadic or continuous two-phase (gas/liquid) flow.
The underlying mechanisms are very similar to the case of batching from empty: the dynamics of gasliquid flow causes high damping. To maintain oscillation a high drive gain is required, and typically at low levels of gas fraction the maximum drive gain of the transmitter is reached, and the flowtube stalls.

The ability of the digital transmitter to maintain oscillation in the presence of two-phase flow has already been demonstrated in Figure 10 (section 5). In lab experiments, it has not been possible to stall a B-tube of any size with any level of gas phase when controlled by the digital transmitter. By contrast, the analogue transmitter stalls with about $2 \%$ gas phase.

Unfortunately, maintaining oscillation is only the first step towards obtaining a satisfactory measurement performance from the flowmeter. Early workers (e.g. Grumski \& Bajura 1984, Sultan \& Hemp, 1989) noted the large mass-flow errors induced in the flowmeter by two-phase processes. A simple model (the 'bubble' model, outlined shortly) was developed to predict the mass-flow error, but poor correlation was found with experimental data, and there has been little further academic consideration of the problem. More recent workers (e.g. Skea \& Hall, 1999), record the effects of two phase flow on later commercial designs, without attempting to model or explain the results.

One difficulty for the early workers was limited knowledge of and access to the transmitter circuitry, and so they had to treat the flowmeter as a black box. In the Oxford programme, the digital transmitter is well understood, and it has been designed to minimise the measurement errors caused by

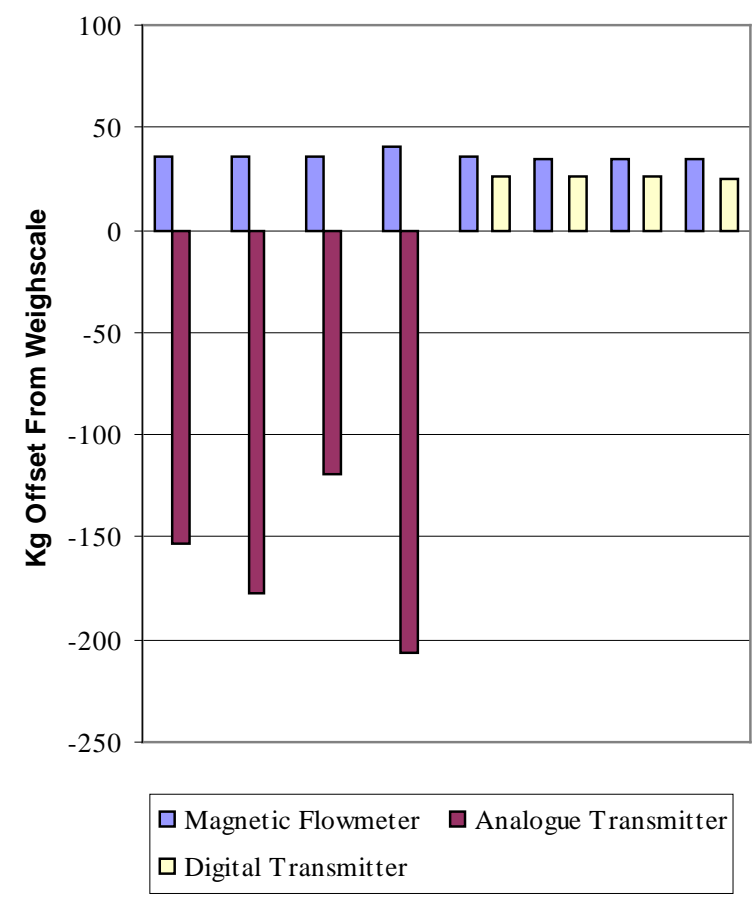

Fig. 19. Results for batching from empty trials in industrial flowlab, 550kg batch size 


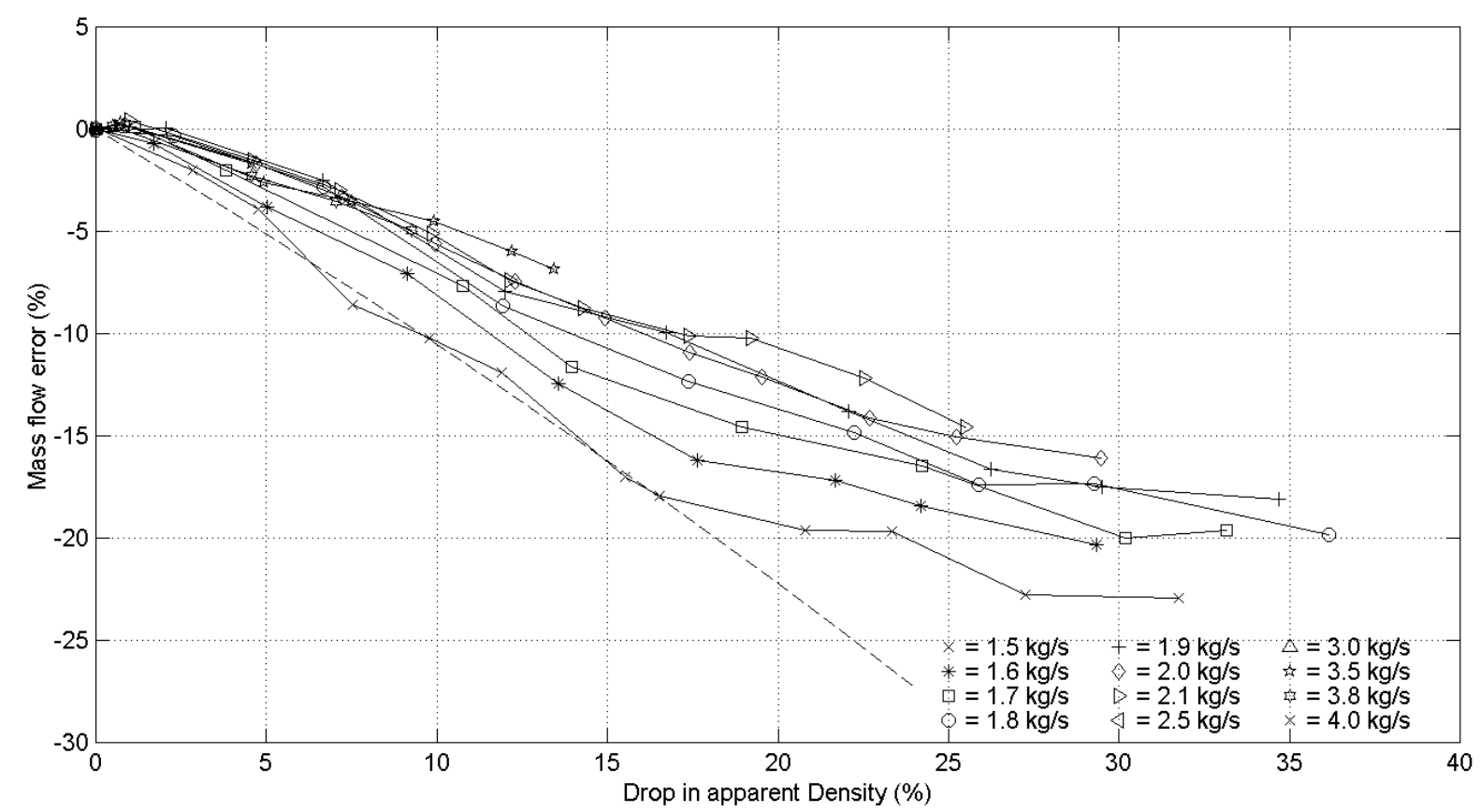

Fig. 20. Mass-flow errors against drop in apparent density, water/air mixture at $22^{\circ} \mathrm{C}$. The dashed line shows the errors predicted by the 'bubble' model

transmitter operation. It is thus the intention that the observed errors are due to the physics of two-phase flow and not to the transmitter response. Certainly for the B-tube, it has been found that the bubble model is a reasonable first approximation to the effect of aeration, and that reasonable on-line correction for two-phase flow is possible.

The 'bubble' or 'effective mass' model was first proposed by Grumski \& Bajura (1984); a detailed description is presented by Sultan (1990). A sphere or bubble of low density (gas) is surrounded by fluid of higher density. If both are subject to acceleration (for example in a vibrating tube), then the bubble moves within the fluid, causing a drop in the observed inertia of the whole system. Defining the void fraction $\alpha$ as the proportion of gas by volume, then the effective mass drops by a proportion $\mathrm{R}$, with

$$
\mathrm{R}=\frac{2 \alpha}{1-\alpha}
$$

Applied to a Coriolis meter, the model predicts that the apparent mass-flow will be less than the true mass-flow by the factor $\mathrm{R}$ as will, by extension, the observed density (not discussed by previous authors). Figure 20 shows the observed mass-flow errors for a series of runs at different flow rates, all using a $25 \mathrm{~mm}$ flowtube in horizontal alignment, and a mixture of water and air at ambient temperature. The $\mathrm{x}$-axis shows the apparent drop in density, rather than the void fraction. It is possible in the laboratory to calculate the void fraction, for example by measuring the gas pressure and flowrate prior to mixing with the fluid, together with the pressure of the two-phase mixture. However, in a plant, only the observed drop in density is available: the true void fraction is not. Note that with the analogue transmitter, air/water mixtures with a density drop of over $5 \%$ cause flowtube stalling so that no data can be collected.

The dashed line shows the relationship between mass-flow error and density drop as predicted by the bubble model. The experimental data follow a similar set of curves, although the model almost always predicts a more negative mass-flow error. Further research is necessary to develop more elaborate models, explaining the discrepancies between the bubble model and data. It is known that the results shown are repeatable, but the importance of other factors, such as the properties of the fluid and gas themselves, is less clear at this stage. However, as shown shortly, it is possible to develop empirical corrections to the mass-flow rate based upon the apparent density as well as several other internally observed variables such as the drive gain and the ratio of sensor voltages.

It is reasonable to assume that the density of the pure fluid is known or can be learned: in many applications the fluid density is relatively constant (particularly if a temperature coefficient can be accommodated within the transmitter software). However, the correction technique used here is not suitable for applications with much variation in the fluid density, or in three-phase flow applications, for which more research would be necessary.

Figure 21 shows the corrected mass-flow measurements. The correction is based on a least squares fit of several internal variables, as well as the bubble model itself. The correction algorithm has only limited applicability, and it is less accurate for lower flow rates (the biggest errors are for 1.5-1.6 $\mathrm{kg} / \mathrm{s}$ ). In horizontal orientation, at lower flow rates the gas and liquid phases begin to separate, and much larger mass-flow errors are observed. Clearly, in 


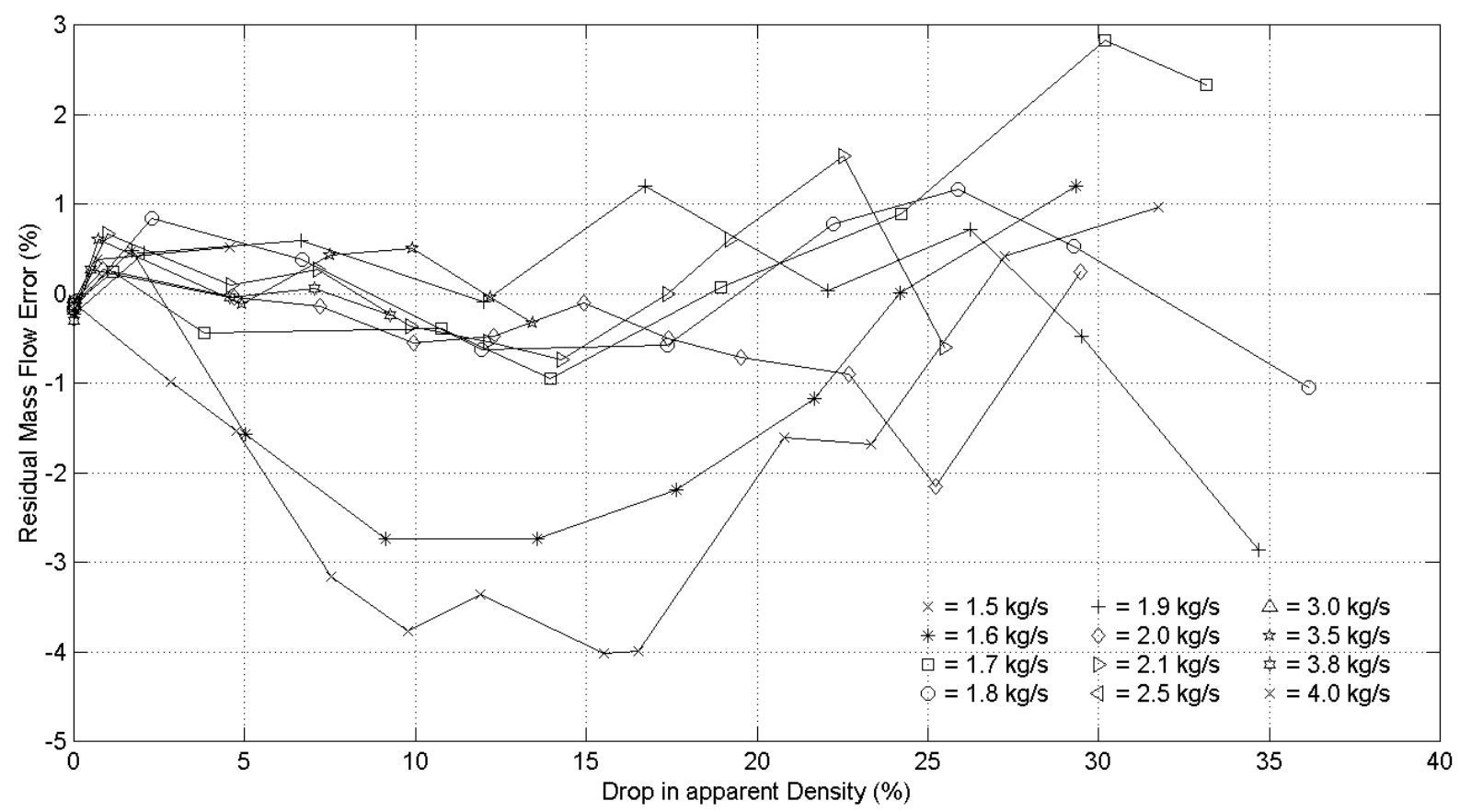

Fig. 21. Residual mass-flow errors after applying corrections based on internally-observed variables

these circumstances the assumptions of the bubble model are no longer valid. However, for higher flow rates the correction is reasonable. During on-line experimental trials a similar correction algorithm has restricted mass flow errors to within about $2.5 \%$ of reading.

For example, Figure 22 shows how the SEVA device responds to the onset of two-phase flow in its reporting of the mass-flow rate (Henry and Clarke, 1993). With single phase flow (up to $t=7 \mathrm{~s}$ ), the massflow measurement is CLEAR and it has a small uncertainty of about $0.2 \%$ of reading. With the onset of two-phase flow, a number of algorithms become active. Firstly, the two phase flow is detected, on the basis of the behaviour of internally observed parameters. Secondly, a measurement correction algorithm is applied, and the measurement is set to BLURRED. Thirdly, the uncertainty of the mass flow increases with the level of void fraction, to a maximum of about $2.3 \%$ of reading. For comparison the uncorrected measurement is also shown. The user thus has the option of continuing operation with the reduced quality of the corrected mass-flow rate, switching to an alternative measurement if available, or shutting down the process.

It is hoped that as the understanding of the effects of two-phase flow improve, better correction techniques can be developed with correspondingly smaller uncertainty bounds, applicable to a broader range of applications.

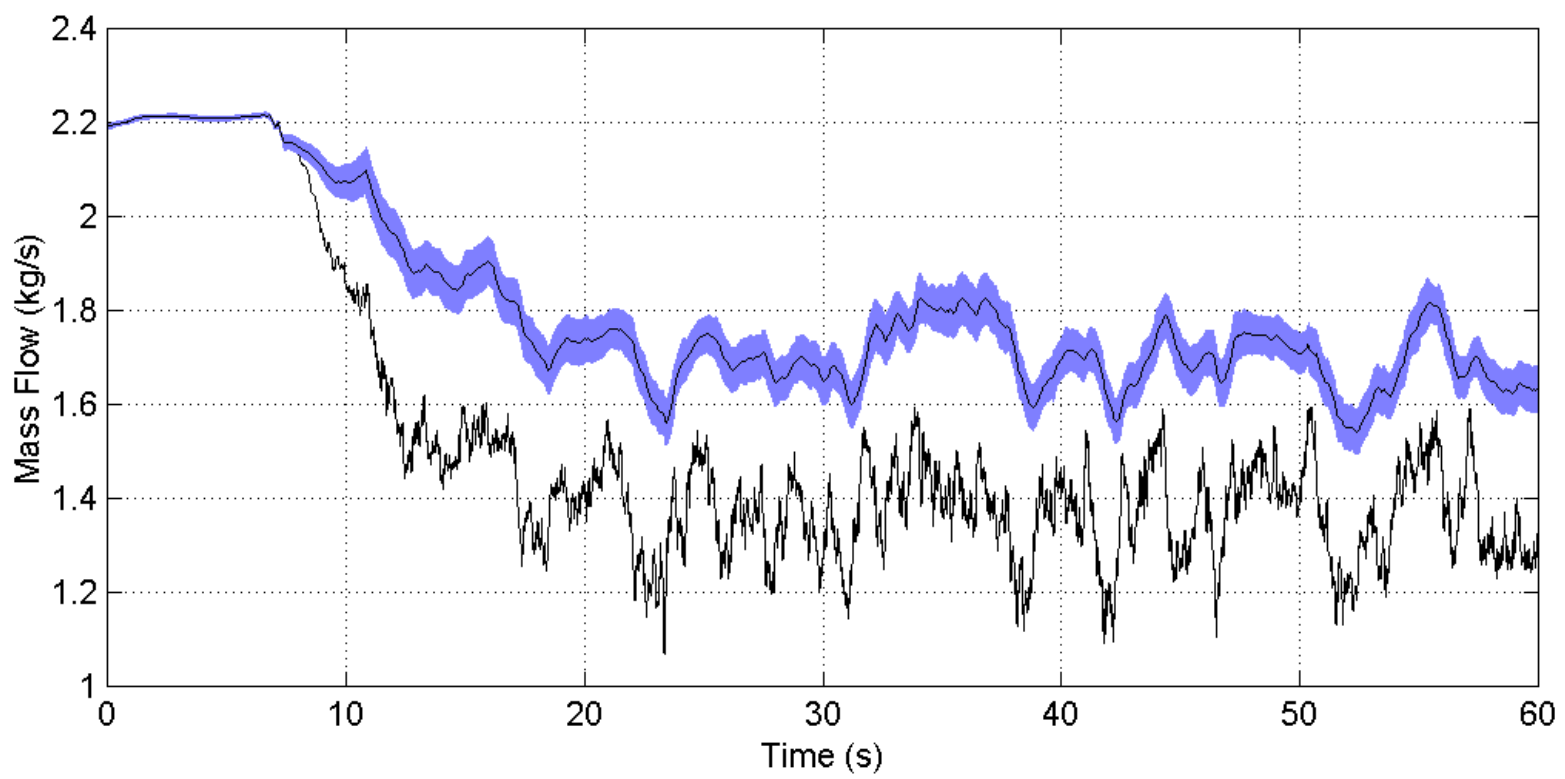

Fig. 22. On-line response of self-validating digital transmitter to the onset of two-phase flow. Lower line: uncorrected mass-flow. Upper line: corrected mass-flow and uncertainty bound. 


\section{Conclusions and further work}

This paper has provided an overview of the digital Coriolis transmitter, describing its background, implementation and examples of its operation. A number of improvements over analogue transmitter performance have been achieved:

- High precision control of flowtube operation, including at very low amplitudes

- The maintenance of flowtube operation even in highly damped conditions

- High precision, high speed measurement

- Compensation for dynamic changes in amplitude

- Compensation for two-phase flow

- Batching to/from empty

This combination of benefits suggests that the digital transmitter represents a significant step forward, not simply a gradual evolution from analogue technology. The ability to deal with two-phase flow and external vibration ensures that the digital transmitter not only gives improved performance in conventional Coriolis applications, but also widens the range of applications to which the flow technology can be applied. The digital platform is furthermore a useful and flexible vehicle for carrying out research into coriolis metering, offering high precision, computing power and data rates.

Prototype transmitters are presently installed in two difficult applications:

- Three meters are installed in a paper mill in Alabama, in a batch application for mixing paper coatings. 25, 50, and $75 \mathrm{~mm}$ meters are used on titanium dioxide, calcium carbonate and clay feed lines respectively. Air entrainment often occurs at the start or end of each batch and between batches. The trial is intended to demonstrate improved repeatability and product consistency through the use of the digital transmitters.

- A 75mm meter has been installed in a feed line to a naptha cracker in Holland. Several products pass through the line, including naptha with a high fraction of $\mathrm{C}_{4}$ gas. The trial will establish whether the digital transmitter is able to perform well in an industrial application with continuous two-phase flow.

These technical advantages have been achieved while at the same time reducing the probable manufacturing costs. This is possible through the use of inexpensive, consumer market components (audio ADCs and DACs, DSPs) a low component count, and implementing a high proportion of functionality in software. The high software content is essential to allow ease of migration to the latest components. For example, virtually every aspect of the digital Coriolis hardware described in this paper is technologically obsolete. The demise of the transputer is well documented, while the 3000 series Xilinx FPGAs and ADC/DAC chips have all been surpassed by one or two generations of products. However, the Handel and $\mathrm{C}$ code are readily migrated to other platforms, for commercialisation or for further research.

This process has already begun. The Handel code has been translated into closely related language Handel$\mathrm{C}$, which is a properly supported commercial product. The Handel code running on 83000 series FPGAs is now executed in a single 4000 series device. The circuitry from three separate Valcard daughtercards has been collected onto a single 3" round card (see Figure 23). New audio components (e.g. a 20-bit integrated codec - Texas Instruments, 1998) will be readily accommodated through FPGA reprogramming.

The prototyping philosophy used here also enables repartitioning of functionality between flowtube and transmitter. For example, it might be considered desirable to develop a flowtube compatible with the new IEEE 1451 smart transducer standard (Costlow, 1998), which defines digital communications between transducer and transmitter with, for example, calibration coefficients stored within transducer registers. Almost certainly the most sensible partition would be to place the entire digital i/o subsystem currently implemented in FPGAs within the flowtube, with a (point-to-point) 1451 interface replacing the Valcard bus shown in Figure 6. Again, the technology suitable for a commercial implementation of such a partition will vary with time, but the core functionality, in terms of software and a netlist of hardware, has been defined.

Future research directions include the following:

- A thorough investigation into the phenomenon of the dynamic measurement effects, including physical modelling, to be extended to the impact of frequency and phase modulation.

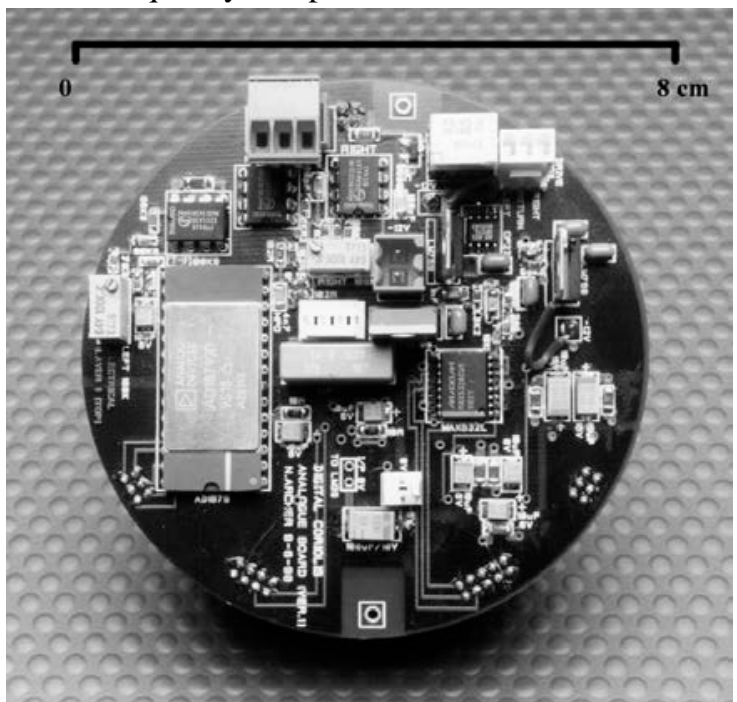

Fig. 23. Round card containing circuitry from three Valcard daughtercards 
- A thorough investigation into the effects of twophase flow, so that compensation can be applied based on a physical understanding of the process.

- Extending the utilisation of FPGAs to include the basic phase, frequency and amplitude calculations, much of which can be implemented in fixed point, dedicated hardware. There will always be the need for a microprocessor to carry out floating point operations such as conversion to engineering units. However, a low power processor surrounded by dedicated hardware in an ASIC (Page, 1998) might, when combined with a flowtube operated at low amplitude, be the basis for a two-wire Coriolis meter.

- The extension of the digital Coriolis transmitter to other flowtube designs. The straight flowtube is considered to have a number of application advantages: low pressure drop, slim profile, while its simple geometry can result in lower manufacturing costs. However, the frequency of oscillation for a straight flowtube can be as high as $1 \mathrm{kHz}$, an order of magnitude greater than the present flowtube. It will be a major challenge to see whether the approach adopted here can be applied to a straight flowtube.

The digital transmitter is seen as the first example of a 'second-generation' SEVA instrument. An inherently unattractive characteristic of faultdetection activity is that it is essentially negative: usually the system under investigation is fixed, and all that can be done is to explore ways in which it can fail. It is far more edifying, where possible, to expend engineering effort in eliminating faults through redesign. While this always entails cost/benefit tradeoffs, today's engineers are fortunate that the digital technology revolution is offering very rapid improvements in the functionality that can be implemented even in low-cost devices. In the case of the digital transmitter, the knowledge gained through validating the current generation of analogue transmitter has led to a much more robust design, reflected in much better performance in difficult applications. This illustrates the view that SEVA promotes a philosophy of continuous measurement quality assessment and improvement, having impact on all aspects of the sensor lifecycle (e.g. design, manufacturing, characterisation, installation, calibration), and not merely on-line operation.

\section{Acknowledgements}

The authors gratefully acknowledge the financial and technical support of the Foxboro Company; technical support from Transtech Ltd and Embedded Solutions Ltd, and EPSRC grants J/44636 (A flexible platform for instrument validation), K/48884 (A Laboratory for Instrument Validation and Digital Communications), K/65867 (A New Technology for
Coriolis Metering - ROPA), and L/41547 (Rapid Implementation of Fieldbus-Compliant, Standalone, Self-Validating Instruments). In addition, the first author is currently supported by an EPSRC Advanced Research Fellowship.

\section{References}

Analog Devices (1998). Details from http://www.analog.com/SHARC/attack.

Bergovic, M., Djuric, P., Dunlap, S. and Phadke, A. (1993). Frequency tracking in power networks in the presence of harmonics. IEEE Transactions on Power Delivery, 8(2), 480-486.

BSI (1998). Meeting of BSI-DISC AMT7 Committee on Fieldbus and Function Blocks, July 1998.

Cascetta, F., Cignolo, G., Goria, R., Martini, G., Rivetti, A. and Vigo, P. (1992). Experimental intercomparision of Coriolis mass-flowmeters. Trans Inst. MC, 14(2), 99-107.

Cheesewright, R. and Clark, C. (1998). The effect of flow pulsations on Coriolis mass-flow meters. Journal of Fluids and Structures, 12, 1025-1039.

Clarke, D.W. (1998). Non-linear control of the oscillation amplitude of a Coriolis mass-flow meter. European Journal of Control. 4(3), 196-207.

Costlow, T. (1998). Smart sensor standard looks for takeoff. EE Times, http://ww.etimes.com/story/OEG19981008s0014.

Grumski, J.T. and Bajura, R.A. (1984). Performance of a Coriolis type mass-flowmeter in the measuring of two-phase (air-liquid) mixtures. ASME Winter Annual Meeting, New Orleans, USA, 17, 9-14.

Henry, M.P. and D.W. Clarke (1993). The selfvalidating sensor: rationale, definitions and examples. Control Engineering Practice, 1(4), 585610.

Henry, M.P. (1994). Validating data from smart sensors. Control Engineering, 41(9), 63-66.

Henry, M.P. (1995a). A self-validating Coriolis meter. Control Engineering, 42(6), 81-86.

Henry, M.P. (1995b). Fieldbus and sensor validation. IEE Computing and Control Journal, 6(6), 263272.

Henry, M.P. (1995c). Keynote Paper: Hardware compilation - a new technique for rapid prototyping of digital systems - applied to sensor validation. Control Engineering Practice, 3(7), 907-924.

Henry M.P., Archer, N., Atia, M.R.A., Bowles, J., Clarke, D.W., Fraher, P.M.A., Page, I., Randall, G., and Yang, J.C-Y. (1996). Programmable hardware architectures for sensor validation. Control Engineering Practice, 4(10), 1339-1354.

Horowitz, P. and Hill, W. (1989). The Art of Electronics $2^{\text {nd }}$ Edition. Cambridge University Press, Cambridge, UK.

Page, I (1998). Hardware compilation, configurable platforms and ASICS for self-validating sensors. hhtp://www.comlab.ox.ac.uk/oucl/hwcomp.html.

Raszillier, H. and Durst, F. (1991). Coriolis-effect in mass-flow metering. Archive of Applied Mechanics, 61, 192-214. 
SIRA (1998). The impact of Fieldbus and sensor fusion on flow measurement. DTI NMS Programme for Flow Measurement, Project OR9A Technical Awareness Studies.

Skea, A.F. and A.R.W. Hall (1999). Effects of gas leaks in oil flow on single-phase flow meters. Flow Measurement and Instrumentation, 10(3), 145-150.

Sultan, G. and J. Hemp (1989). Modelling of the Coriolis mass-flowmeter. J. Sound and Vib., 132(3), 473-489.

Sultan, G. (1990) Theoretical and experimental studies of the Coriolis mass-flowmeter. PhD thesis Cranfield University, UK.

Texas Instruments (1998). http:/www.ti.com; search for TLC320AD75. 